\title{
Slowly rotating black holes in Einsteinian cubic gravity
}

\author{
Connor Adair, ${ }^{*}$ Pablo Bueno, ${ }^{\star}$ Pablo A. Cano, ${ }^{\uparrow}$ Robie A. Hennigar ${ }^{\mathbb{4}}$ and Robert B. Mann ${ }^{*}$ \\ * Department of Physics and Astronomy, University of Waterloo \\ Waterloo, Ontario, Canada, N2L $3 G 1$ \\ * Instituto Balseiro, Centro Atómico Bariloche \\ 8400-S.C. de Bariloche, Río Negro, Argentina \\ ₹ Instituut voor Theoretische Fysica, KU Leuven \\ Celestijnenlaan 200D, B-3001 Leuven, Belgium \\ 4I Department of Mathematics and Statistics, Memorial University of Newfoundland \\ St. John's, Newfoundland and Labrador, A1C 5S7, Canada
}

\begin{abstract}
We construct slowly rotating black-hole solutions of Einsteinian cubic gravity (ECG) in four dimensions with flat and AdS asymptotes. At leading order in the rotation parameter, the only modification with respect to the static case is the appearance of a non-vanishing $g_{t \phi}$ component. Similarly to the static case, the order of the equation determining such component can be reduced twice, giving rise to a second-order differential equation which can be easily solved numerically as a function of the ECG coupling. We study how various physical properties of the solutions are modified with respect to the Einstein gravity case, including its angular velocity, photon sphere, photon rings, shadow, and innermost stable circular orbits (in the case of timelike geodesics).
\end{abstract}




\section{Contents}

1 Introduction $\quad 1$

2 Asymptotically flat solutions $\quad 3$

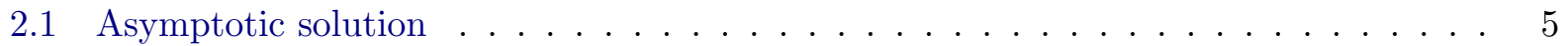

2.2 Near horizon solution $\ldots \ldots \ldots \ldots \ldots \ldots$

2.3 Numerical solution . . . . . . . . . . . . . . . . . . . . . 8

3 Properties of the solution $\quad 11$

3.1 Angular velocity of the horizon . . . . . . . . . . . . . . . . . 12

3.2 Geodesics . . . . . . . . . . . . . . . . . . . . 13

3.3 Geodesics in the equatorial plane . . . . . . . . . . . . . . . 15

3.4 Black hole shadow . . . . . . . . . . . . . . . . . . . . . . 21

4 Asymptotically AdS solutions 23

4.1 Asymptotic solution . . . . . . . . . . . . . . . . . . . 24

4.2 Near horizon solution . . . . . . . . . . . . . . . . 25

4.3 Numerical solution . . . . . . . . . . . . . . . . . . . . . 26

4.4 Spinning solutions in the critical limit . . . . . . . . . . . . . 28

5 Final comments $\quad 30$

A Slowly rotating black holes in Lovelock gravities $\quad 31$

A.1 Einstein gravity . . . . . . . . . . . . . . . . . . . . 31

A.2 Lovelock gravities . . . . . . . . . . . . . . . . . . . . . 33

\section{Introduction}

Constructing black hole solutions of higher-curvature modifications of Einstein gravity is a challenging task. Restricting the discussion to pure-metric diffeomorphism-covariant theories, even the simplest case of static and spherically symmetric configurations is very difficult to tackle in general, typically involving fourth-order coupled differential equations.

Nonetheless, there are exceptions to this. The prototypical example corresponds to Lovelock theories [1,2], for which (analytic) generalizations of the usual Schwarzschild solution were constructed long ago [3-6] and more recently [7-14]. Naturally, such solutions are nontrivial only for $D \geq 5$, as all higher-curvature Lovelock densities are either topological or trivial in $D=4$. Also for $D \geq 5$, there exists another class of theories which admit analytic static black holes. These are the so-called Quasi-topological gravities [15-20]. The static black hole solutions of Quasi-topological gravities are characterized by a single function which, similarly to what happens for Lovelock theories, is determined by an algebraic equation. ${ }^{1}$ Recently, it has been realized that both Lovelock and

\footnotetext{
${ }^{1}$ It should be noted that for the case of Lovelock gravity, the equations of motion are algebraic under more general
} 
Quasi-topological theories can be thought of as particular cases of a more general type of theories, the so-called Generalized Quasi-topological (GQT) gravities [22-26]. These are characterized by admitting single-function $\left(g_{t t} g_{r r}=-1\right)$ non-hairy generalizations of the Schwarzschild black hole as well as by possessing second-order linearized equations around maximally symmetric backgrounds. A detailed list of their properties have been given [26, 27], and the thermodynamics of the cubic and quartic cases have been studied in detail [25, 28-30]; see [31-33] for related references. In GQT theories, the equation that determines the black hole metric function $f(r) \equiv-g_{t t}$ can be automatically integrated once [23], and we can distinguish two cases. The first group corresponds to theories for which the resulting equation is algebraic, whereas the second includes theories for which it is a second-order differential equation. Lovelock and Quasi-topological gravities comprise the first group.

The first representative of the second group to be identified was Einsteinian cubic gravity (ECG). This theory was originally constructed as the most-general higher-curvature modification of Einstein gravity which, up to cubic order, only propagates the usual transverse and traceless graviton on maximally symmetric backgrounds in general dimensions [34]. Soon after, it was realized that analogously to Lovelock and Quasi-topological theories in $D \geq 5$ - four-dimensional ECG admits non-hairy single-function generalizations of the Schwarzschild black hole [35, 36]. The action of the theory is given by

$$
I_{\mathrm{ECG}}=\frac{1}{16 \pi G} \int d^{4} x \sqrt{-g}\left[R-\frac{\mu L^{4}}{8} \mathcal{P}\right],
$$

where $\mu$ is a dimensionless coupling, $L$ some length scale and $\mathcal{P}$ is given by [34]

$$
\mathcal{P}=12 R_{a b}^{c d} R_{c d}^{e f} R_{e f}^{a b}+R_{a b}^{c d} R_{c d}^{e f} R_{e f}^{a b}-12 R_{a b c d} R^{a c} R^{b d}+8 R_{a}^{b} R_{b}^{c} R_{c}^{a} .
$$

The construction of ECG opened the door to the definition and classification of GQT gravities, which are now known to exist at general orders and in arbitrary dimensions [26]. The static black hole solutions of ECG have been by now studied in numerous papers and in several contexts [37$46 .^{2}$

Besides ECG and its higher-order cousins, there exist a few other static black hole solutions of higher-cuvature modifications of Einstein gravity in $D=4$. An important set of such solutions (for which $g_{t t} g_{r r} \neq 1$ ) was presented in [56, 57] for quadratic modifications of Einstein gravity. More solutions are available for the so-called "non-polynomial" gravities — see e.g., [58, 59] and refs. therein.

All we have said so far concerns static black holes. When rotation is included, things become considerably more involved. For instance, no analytic generalization of the higher-dimensional Kerr (or Myers-Perry [60]) solution has been constructed so far for Lovelock theories in the case of arbitrary rotation. In fact, it has been argued that the Kerr-Schild ansatz does not help in that case [61] (at least in the Einstein-Gauss-Bonnet case). Only for certain values of the Lovelock couplings $[61,62]$ or in the slowly rotating limit [63-65] explicit solutions have been constructed, though full-fledged solutions have been numerically obtained [66,67]. Additional relevant approaches to higher-curvature modifications of the Kerr solution in $D=4$ include the construction of solutions at leading order in the effective gravitational action couplings [68-70].

circumstances - see [21]. While to the best of our knowledge similar proofs are lacking for quasi-topological gravities, we expect the analogous results will not hold generally. It would be worthwhile to explore this question in greater detail.

${ }^{2}$ Cosmological applications of the theory have been also explored [47-55]. 
As far as ECG is concerned, near-horizon rotating solutions have been constructed in [71] and perturbative solutions in the gravitational coupling have been obtained in [72]. In the present paper we construct the slowly rotating black holes of the theory. The metric (assuming the condition of axisymmetry) takes the form

$$
d s^{2}=-f(r) d t^{2}+\frac{d r^{2}}{f(r)}+2 a r^{2} p(r) \sin ^{2} \theta d t d \phi+r^{2}\left[d \theta^{2}+\sin ^{2} \theta d \phi^{2}\right] .
$$

With respect to the static case, fully characterized by the function $f(r)$, the only modification is the appearance of a nonvanishing $g_{t \phi}$ component. Similarly to what happens for general GQT gravities in the static case - as well as for Taub-NUT/Bolt solutions [73] — we observe that the order of the equation that determines the function $p(r)$ can be reduced twice, leaving us with a second-order differential equation that can be easily handled and solved numerically. This allows us to explicitly perform numerous computations of physically relevant quantities of the solution.

Besides theoretical considerations, the birth of the gravitational wave-astronomy era [74] should eventually allow for a detailed scrutiny of the validity of the Kerr solution as the putative description of astrophysical black holes. This further motivates the study of rotating black hole solutions and their physical properties in modified theories of gravity.

The structure of our paper is as follows. In Section 2 we construct the asymptotically flat slowly rotating black hole of four-dimensional ECG. As anticipated above, we show that the only new equation to be solved is a second-order differential equation. We analyze the asymptotic and near-horizon solutions, and then we construct the full solutions numerically. For a given value of the ECG coupling, each solution is fully characterized by its mass and angular momentum. In Section 3 we study geodesics in the new slowly rotating black hole background and how they differ from their slowly rotating Kerr black hole counterparts. In the case of null geodesics, we study the photon sphere, the photon rings (and the Lyapunov exponents controlling their instability) and the black hole shadow as seen by an asymptotic observer. For timelike geodesics, we compute how the innermost stable circular orbit is modified. We also compute the horizon angular velocity here. In Section 4 we repeat the analysis of Section 2 in the presence of a negative cosmological constant and construct the corresponding asymptotically AdS solutions. We also construct a new analytic rotating solution in the critical limit of the theory. Section 5 contains some final comments regarding possible future studies. Appendix A contains a review of the slowly rotating black holes (with a single axis of rotation) of Einstein gravity and general Lovelock theories in arbitrary dimensions.

\section{Asymptotically flat solutions}

The equations of motion of ECG are given by [22, 34]

$$
P_{a c d e} R_{b}^{c d e}-\frac{1}{2} g_{a b} \mathcal{L}_{\mathrm{ECG}}-2 \nabla^{c} \nabla^{d} P_{a c d b}=0, \quad P_{a b c d} \equiv \frac{\partial \mathcal{L}_{\mathrm{ECG}}}{\partial R^{a b c d}},
$$

where

$$
\begin{aligned}
P_{a b c d} & =\frac{1}{16 \pi G}\left[g_{a[c} g_{b] d}-\frac{3 \mu L^{4}}{4}\left[R_{a d} R_{b c}-R_{a c} R_{b d}+g_{b d} R_{a}{ }^{e} R_{c e}-g_{a d} R_{b}{ }^{e} R_{c e}-g_{b c} R_{a}{ }^{e} R_{d e}\right.\right. \\
& +g_{a c} R_{b}^{e} R_{d e}-g_{b d} R^{e f} R_{a e c f}+g_{b c} R^{e f} R_{a e d f}+g_{a d} R^{e f} R_{b e c f}-3 R_{a}{ }^{e}{ }_{d}^{f} R_{b e c f}
\end{aligned}
$$




$$
\left.\left.-g_{a c} R^{e f} R_{b e d f}+3 R_{a}{ }^{e f} R_{b e d f}+\frac{1}{2} R_{a b}{ }^{e f} R_{c d e f}\right]\right] .
$$

We shall write the ansatz (3) for the slowly rotating black hole in the following form

$$
d s^{2}=-N(r)^{2} f(r) d t^{2}+\frac{d r^{2}}{f(r)}+2 a r^{2} p(r)\left(1-x^{2}\right) d t d \phi+r^{2}\left[\frac{d x^{2}}{1-x^{2}}+\left(1-x^{2}\right) d \phi^{2}\right],
$$

where $x \equiv \cos \theta$. Note that this includes three-independent functions of the radial coordinate. We wish to solve the field equations (4) for this metric ansatz, working at linear order in $a$. At this order, only $\mathcal{E}_{t \phi}$ becomes modified. The $\mathcal{E}_{t t}$ and $\mathcal{E}_{r r}$ are identical to the static case, for which $\mathcal{E}_{t}{ }^{t}=\mathcal{E}_{r}{ }^{r}$. It follows that this relation also holds in the slowly rotating case, which implies that we can set $N(r)=1$ without loss of generality.

This $\mathcal{E}_{t \phi}$ component of the field equations is a complicated, fourth-order, differential equation in the function $p(r)$. However the following combination of the components of the field equations

$$
\frac{r^{4}}{f(r)}\left[\mathcal{E}_{t}{ }^{\phi}-\frac{\operatorname{arp}(r)}{2} \frac{d \mathcal{E}_{r}{ }^{r}}{d r}\right]
$$

remarkably admits a trivial first integral. The resulting third-order equation takes the form

$$
\begin{aligned}
C & =r^{4} p^{\prime}+\mu\left[-\frac{3}{2}\left(\frac{r f^{\prime}}{2}+1-f\right) r^{2} f p^{\prime \prime \prime}-\frac{3}{2}\left(\frac{r^{2} f}{2} f^{\prime \prime}+\frac{r^{2} f^{\prime 2}}{2}+\frac{r(2-f) f^{\prime}}{2}+2 f(1-f)\right) p^{\prime \prime}\right. \\
& \left.+\frac{15}{2}\left(-\frac{3 r^{2}}{10}\left(-\frac{r f^{\prime}}{3}+1+\frac{2 f}{3}\right) f^{\prime \prime}-\frac{r^{2} f^{\prime 2}}{2}+\frac{r(1+7 f) f^{\prime}}{5}+(1-f)\left(1+\frac{6}{5} f\right)\right) p^{\prime}\right],
\end{aligned}
$$

with $C$ a constant of integration, which we show below to be proportional to the mass of the solution through $C=6 \mathrm{M}$. We note that a second remarkable thing has occurred upon integration: while the equation for $p(r)$ is now a third-order equation, there is no term proportional to $p(r)$ itself. This means that we can further simplify the problem by defining $p(r)=\int^{r} g\left(r^{\prime}\right) d r^{\prime}$ which gives a second-order differential equation for the unknown function $g(r)$. We emphasize that these simplifying features would not hold for general gravity theories - they are a result of the special properties of (a subset of) generalized quasi-topological gravities.

We therefore must solve the following two equations to determine the two unknown metric functions:

$$
\begin{aligned}
2 G M & =r(1-f)+\frac{\mu L^{4}}{4 r^{2}}\left[6 r\left(\frac{r f^{\prime}}{2}+1-f\right) f^{\prime \prime} f-\left(r^{2} f^{\prime 2}+3 r f^{\prime}+6 f(1-f)\right) f^{\prime}\right], \\
C & =r^{4} g-\frac{3 \mu L^{4}}{2}\left[\left(\frac{r f^{\prime}}{2}+1-f\right) r^{2} f g^{\prime \prime}+\left(\frac{r^{2} f}{2} f^{\prime \prime}+\frac{r^{2} f^{\prime 2}}{2}+\frac{r(2-f) f^{\prime}}{2}+2 f(1-f)\right) g^{\prime}\right. \\
& \left.-5\left(-\frac{3 r^{2}}{10}\left(-\frac{r f^{\prime}}{3}+1+\frac{2 f}{3}\right) f^{\prime \prime}-\frac{r^{2} f^{\prime 2}}{2}+\frac{r(1+7 f) f^{\prime}}{5}+(1-f)\left(1+\frac{6}{5} f\right)\right) g\right] .
\end{aligned}
$$

The first of these equations is the usual (integrated) $\mathcal{E}_{r}{ }^{r}$ component of the field equations for a static black hole ansatz that has been studied in the context of four-dimensional spherically symmetric solutions [35, 36], while the second equation will account for the new physics due to the rotation. We would like to stress that this double order-reduction phenomenon that allows us to reduce the problem to two second-order differential equations for $f(r)$ and $g(r)$ — which, besides, are linear in $f^{\prime \prime}(r)$ and $g^{\prime \prime}(r)$ respectively — is highly nontrivial. It would be interesting to explore whether 
this extends to general GQTGs in $D=4$ (and also in higher dimensions, possibly with several independent rotation parameters turned on).

In the weak coupling limit, we can solve the equations above by assuming a perturbative expansion in $\mu$ of the functions $f$ and $g$. This process leads to the following solution up to order $\mu^{2}$ :

$$
\begin{aligned}
f(r) & =1-\frac{2 M}{r}+\mu L^{4}\left[-\frac{27 M^{2}}{r^{6}}+\frac{46 M^{3}}{r^{7}}\right]-\left(\mu L^{4}\right)^{2}\left[\frac{6804 M^{3}}{r^{11}}-\frac{27702 M^{4}}{r^{12}}+\frac{28014 M^{5}}{r^{13}}\right], \\
r^{2} p(r) & =-\frac{2 M}{r}+\frac{46 \mu L^{4} M^{3}}{r^{7}}+\left(\mu L^{4}\right)^{2}\left[\frac{4860 L^{8} M^{3}}{13 r^{11}}+\frac{12393 L^{8} M^{4}}{r^{12}}-\frac{28014 L^{8} M^{5}}{r^{13}}\right] .
\end{aligned}
$$

However, this solution is only valid for rather small values of $\mu L^{4} / M^{4}$, and adding more terms in the expansion does not increase the precision. ${ }^{3}$ Thus, we need to perform a non-perturbative analysis in order to understand the solutions at large coupling. Let us then study the solutions to the equations (9) and (10) in more detail.

\subsection{Asymptotic solution}

The solution of the equation for $f(r)$ has been quite thoroughly studied in other work - see, e.g., $[22,25,35,38,39]$ — so here we will be relatively concise with the analysis.

Asymptotically, a power series solution of the equations of motion can be easily obtained. Taking as an ansatz

$$
f_{1 / r}(r)=\sum_{n=0} \frac{b_{n}}{r^{n}}
$$

plugging this into the equations of motion and solving order-by-order in the large- $r$ limit we obtain the following:

$$
f_{1 / r}=1-\frac{2 M}{r}-\frac{27 M^{2} \mu L^{4}}{r^{6}}+\mathcal{O}\left(r^{-7}\right) .
$$

However, this form of the solution could be neglecting effects non-perturbative in the coupling or terms that vanish non-polynomially fast as $r \rightarrow \infty$. To gain an understanding of these possible corrections and their implications, we add to the power series solution an arbitrary correction $f_{\mathrm{h}}(r)$ and plug $f(r)=f_{1 / r}+f_{\mathrm{h}}(r)$ back into (9), working to linear order in $f_{\mathrm{h}}(r)$. This yields a homogeneous equation for the correction $f_{\mathrm{h}}(r)$ :

$$
-\frac{9 M L^{4} \mu}{2} f_{\mathrm{h}}^{\prime \prime}(r)+r^{3} f_{\mathrm{h}}(r)=0
$$

where we have kept only the leading terms in the large- $r$ limit. ${ }^{4}$ The equation for the homogeneous

\footnotetext{
${ }^{3}$ In fact, an analysis of the series in $\mu$ suggests that its radius of convergence is zero. Nevertheless, in the sense of asymptotic series, the perturbative expansion with a few terms does provide a reasonable approximation for small enough coupling.

${ }^{4}$ This means, for example, that we are neglecting the term proportional to $f_{\mathrm{h}}^{\prime}$, which falls off faster than the other terms. Working to higher-order and including this term does not change the conclusions.
} 
solution admits a solution in terms of special functions,

$$
f_{\mathrm{h}}(r) \sim A \sqrt{r} I_{1 / 5}\left(\frac{2 \sqrt{2} r^{5 / 2}}{15 \sqrt{M \mu L^{4}}}\right)+B \sqrt{r} K_{1 / 5}\left(-\frac{2 \sqrt{2} r^{5 / 2}}{15 \sqrt{M \mu L^{4}}}\right),
$$

where $A$ and $B$ are constants and $K_{\nu}(x)$ and $I_{\nu}(x)$ are Bessel functions. We see that the requirement of a well-behaved positive mass solutions fixes the parameter $A=0$ and demands $\mu>0$. The second part of the solution, which decays super-exponentially, can be safely neglected when writing the asymptotic solution.

We now turn to an analysis of eq. (10). The method of solution is the same as for $f(r)$ : we first obtain a power-series solution, and then seek to understand possible corrections to this. The series solution is easily obtained by solving the field equations in the large- $r$ limit, and reads:

$$
g_{1 / r}(r)=\frac{C}{r^{4}}-\frac{69 C M \mu L^{4}}{r^{10}}+\mathcal{O}\left(r^{-12}\right) .
$$

The homogeneous equation can be obtained by substituting $g(r)=g_{1 / r}(r)+g_{\mathrm{h}}(r)$ back into (10) and keeping only the most important terms in the large- $r$ limit. This yields the following equation:

$$
-\frac{9 M \mu L^{4}}{2} g_{\mathrm{h}}^{\prime \prime}(r)+r^{3} g_{\mathrm{h}}(r)=0 .
$$

The equation is of exactly the same form as that presented above in (16) for $f_{\mathrm{h}}$, and so the basic conclusion is the same: we must fix one integration constant to zero (the coefficient of the growing mode) and restrict the coupling to positive values in order to have well-behaved positive mass solutions.

The equation that determines $p(r)$ was originally fourth-order and, as a result, the solution should be characterized by four parameters. We see that three of these parameters arise as integration constants in the solution for $g(r)$ : the constant $C$ and two more constants of integration in the homogeneous solution. One of these latter parameters - the coefficient of the growing mode - is fixed to zero by requiring well-behaved asymptotics. We will see in the following subsections that a free parameter in the near-horizon solution is fixed by requiring that the near horizon solution connects smoothly to the asymptotic solution. Let us now discuss the interpretation of the integration constant $C$ and the final parameter that arises when integrating $g(r)$ to obtain $p(r)$.

In the large- $r$ limit, the behaviour of $p(r)$ is accurately described by integrating just the particular solution $g_{1 / r}(r)$. This gives

$$
p(r)=-\frac{\Omega_{\infty}}{a}-\frac{C}{3 r^{3}}+\frac{23 C \mu M^{2} L^{4}}{3 r^{9}}+\mathcal{O}\left(r^{-11}\right),
$$

where $\Omega_{\infty}$ is a constant of integration. It is easy to see that $\Omega_{\infty}$ is related to the asymptotic angular velocity of the spacetime. We have

$$
\Omega=-\frac{g_{t \phi}}{g_{\phi \phi}} \rightarrow-a \lim _{r \rightarrow \infty} p(r)=\Omega_{\infty} .
$$

By a suitable choice of the Killing coordinates $t$ and $\phi$ it is always possible to set $\Omega_{\infty}=0$, and so henceforth we will make this choice. This amounts to starting the integration for $p(r)$ at $r=\infty$ and working inward. 
To interpret the integration constant $C$, we will require that our solution asymptotically approaches the slow rotation limit of the Kerr solution

$$
d s^{2} \rightarrow-\left(1-\frac{2 M}{r}\right) d t^{2}-\frac{4 M a\left(1-x^{2}\right)}{r} d t d \phi+\frac{d r^{2}}{\left(1-\frac{2 M}{r}\right)}+r^{2}\left[\frac{d x^{2}}{1-x^{2}}+\left(1-x^{2}\right) d \phi^{2}\right] .
$$

Comparing this with our asymptotic expansion for $p(r)$ reveals that

$$
C=6 M
$$

This ensures that the spin parameter $a=J / M$ represents the angular momentum per unit mass of our solution.

\subsection{Near horizon solution}

We now wish to consider the equations of motion expanded about the horizon of a black hole. Within the first-order slow rotation approximation there is no displacement of the horizon and it remains located at $f\left(r_{\mathrm{h}}\right)=0$. Let us first discuss the near horizon solution for the function $f(r)$. In this case, since we wish to study black hole solutions, we will assume that $f(r)$ goes to zero linearly as $r \rightarrow r_{\mathrm{h}}$ :

$$
f(r)=4 \pi T\left(r-r_{\mathrm{h}}\right)+\sum_{n=2} a_{n}\left(r-r_{\mathrm{h}}\right)^{n} .
$$

Plugging this ansatz into the first equation of (9) and expanding order by order in $\left(r-r_{\mathrm{h}}\right)$, we find that the first two relationships can be expressed as

$$
\begin{aligned}
2 G M & =r_{\mathrm{h}}-\frac{3(4 \pi T)^{2} \mu L^{4}}{4 r_{\mathrm{h}}}-\frac{(4 \pi T)^{3} \mu L^{4}}{4}, \\
0 & =-1+4 \pi T r_{\mathrm{h}}+\frac{3(4 \pi T)^{2} \mu L^{4}}{4 r_{\mathrm{h}}^{2}} .
\end{aligned}
$$

These two conditions suffice to determine the horizon radius and temperature of the black hole for a given choice of the mass and coupling. At the next order, $a_{3}$ appears linearly and can be solved for in terms of the parameter $a_{2}$, along with the mass and horizon radius. The general pattern is that $a_{n}$ for $n>2$ can be solved for in terms of the preceding parameters, which can themselves be expressed in terms of $a_{2}$, the mass and the coupling.

The parameter $a_{2}$ is not fixed by the near-horizon equations of motion. However, as we will see explicitly when considering the numerical solution, the value of $a_{2}$ is fixed by requiring the solution joins smoothly on to the asymptotic solution $[25,38]$, we will refer to this special value as $a_{2}^{\star}$. An alternative prescription to determine $a_{2}^{\star}$ involves fixing the $\mu$-derivatives of $a_{2}$ by the requirement that the near horizon solution admits a smooth $\mu \rightarrow 0$ limit — we refer the reader to [38, 39] for a more detailed discussion of this technique. The basic idea is to remove any terms proportional to inverse powers of $\mu$ in the series expansion of the term $a_{n}$ for small $\mu$. This, in fact, fixes all the derivatives of $a_{2}$ with the first few of these reading:

$$
a_{2}^{(0)}=-\frac{1}{r_{\mathrm{h}}^{2}}, \quad a_{2}^{(1)}=\frac{81 L^{4}}{4 r_{\mathrm{h}}^{6}}, \quad a_{2}^{(2)}=-\frac{3807 L^{8}}{2 r_{\mathrm{h}}^{10}}, \quad a_{2}^{(3)}=\frac{17827209 L^{12}}{32 r_{\mathrm{h}}^{14}},
$$


where $a_{2}^{(n)}:=\left.\left[(d / d \mu)^{n} a_{2}(\mu)\right]\right|_{\mu=0}$. The coefficients grow faster than $n$ ! and a convergence analysis reveals that the power series expansion has vanishing radius of convergence. Thus, regarded as a function of the coupling, $a_{2}$ is a smooth but non-analytic function. However, as was discussed in [38], using a Padé approximant to represent $a_{2}(\mu)$ yields a result that matches quite well the numerically determined value of $a_{2}^{\star}$.

We do not expect that the function $g(r)$ should vanish at the horizon, but we do expect that it is a smooth function there. Therefore, we take the following series ansatz

$$
g(r)=\sum_{n=0} g_{n}\left(r-r_{\mathrm{h}}\right)^{n} .
$$

Substituting this ansatz, along with the near horizon expansion for $f(r)$, into eq. (10) we can solve order by order for the parameters $g_{n}$. In this case, we find that all of the parameters $g_{n}$ for $n>0$ can be obtained explicitly in terms of the mass, the coupling, and the parameters $a_{2}$ and $g_{0}$. The first few relationships read:

$$
\begin{aligned}
6 M & =g_{0}\left(\frac{3}{4} \mu L^{4}\left(2 r_{\mathrm{h}}\left[(4 \pi T)-3 a_{2} r_{\mathrm{h}}\right]+(4 \pi T) r_{\mathrm{h}}^{2}\left[2 a_{2} r_{\mathrm{h}}-5(4 \pi T)\right]+10\right)+r_{\mathrm{h}}^{4}\right) \\
& -\frac{3}{4}(4 \pi T) g_{1} \mu L^{4} r_{\mathrm{h}}\left[(4 \pi T) r_{\mathrm{h}}+2\right] \\
0 & =g_{1}\left(\frac{3}{4} \mu L^{4}\left(-2 r_{\mathrm{h}}\left[5 a_{2} r_{\mathrm{h}}+3(4 \pi T)\right]-(4 \pi T) r_{\mathrm{h}}^{2}\left[4 a_{2} r_{\mathrm{h}}+7(4 \pi T)\right]+10\right)+r_{\mathrm{h}}^{4}\right) \\
& +g_{0}\left(\frac{3}{2} \mu L^{4}\left(\left[2(4 \pi T)-r_{\mathrm{h}}\left(9 a_{3} r_{\mathrm{h}}+4 a_{2}\right)\right]+r_{\mathrm{h}}\left[2 a_{2}^{2} r_{\mathrm{h}}^{2}+3(4 \pi T) r_{\mathrm{h}}\left(a_{3} r_{\mathrm{h}}-3 a_{2}\right)+2(4 \pi T)^{2}\right]\right)\right. \\
& \left.+4 r_{\mathrm{h}}^{3}\right)-3(4 \pi T) g_{2} \mu L^{4} r_{\mathrm{h}}^{2}\left[(4 \pi T) r_{\mathrm{h}}+2\right] .
\end{aligned}
$$

The situation for $g_{0}$ is quite similar to the situation for $a_{2}$. While it appears as a free parameter in the near horizon expansion, a numerical analysis reveals that the solution for $g(r)$ only joins smoothly onto the asymptotic solution if the parameter $g_{0}$ takes on a special value, which we will denote as $g_{0}^{\star}$. Again, if we demand a smooth $\mu \rightarrow 0$ limit of the near horizon solution this fixes all of the derivatives of $g_{0}^{\star}$, with the first few reading

$$
g_{0}^{(0)}=\frac{3}{r_{\mathrm{h}}^{3}}, \quad g_{0}^{(1)}=-\frac{219 L^{4}}{4 r_{\mathrm{h}}^{7}}, \quad g_{0}^{(2)}=\frac{3681 L^{4}}{r_{\mathrm{h}}^{11}}, \quad g_{0}^{(3)}=-\frac{22862007 L^{12}}{32 r_{\mathrm{h}}^{15}},
$$

where $\left.g_{0}^{(n)} \equiv\left[(d / d \mu)^{n} g_{0}(\mu)\right]\right|_{\mu=0}$. Once again, due to the growth of the coefficients, a power series for $g_{0}$ based on this expansion does not converge - it is a smooth but non-analytic function of the coupling $\mu$.

\subsection{Numerical solution}

Let us now construct the full solution numerically. We start again by discussing the construction of the numerical solution for $f(r)$. This has been discussed previously e.g., [25], but we include the details here for completeness, since the situation for $g(r)$ is very similar.

The basic idea here is to use the near horizon expansion constructed in Section 2.2 to generate initial data to be used in a numerical solver that integrates from the horizon toward infinity (or 

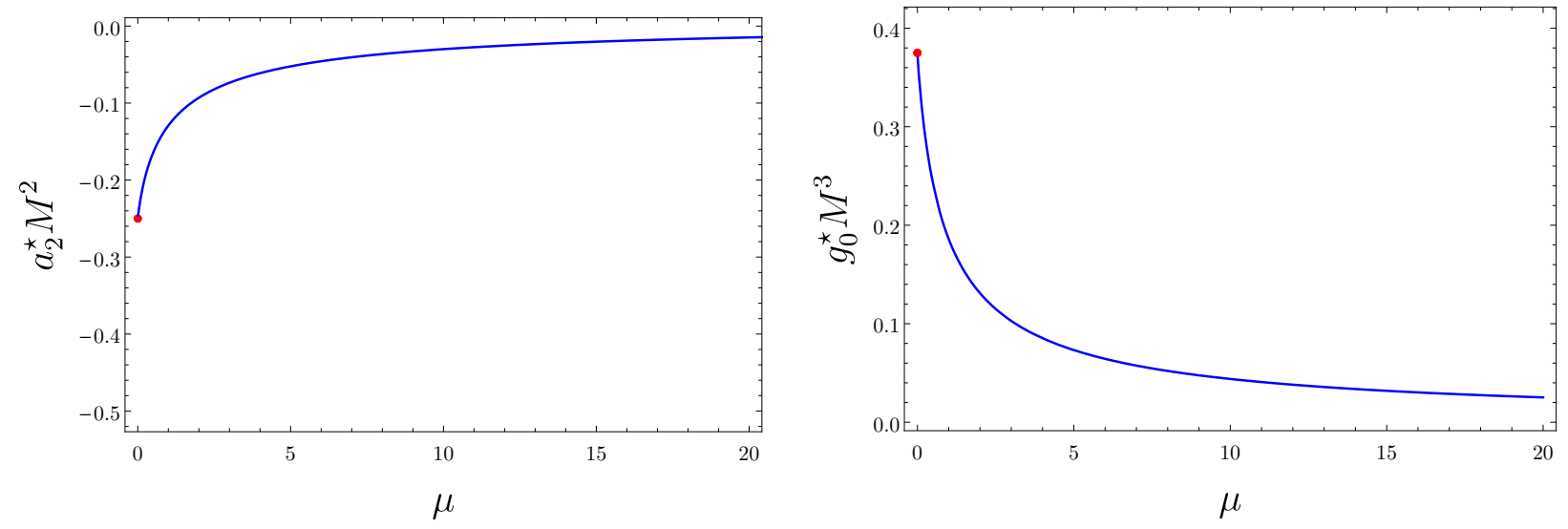

Figure 1: Here we show an interpolation of the numerically determined value of $a_{2}^{\star}$ (left) and $g_{0}^{\star}$ (right) as functions of the higher-curvature coupling $\mu$. The red dots indicate the values for the Einstein gravity solution, which read: $a_{2}=-1 /\left(4 M^{2}\right)$ and $g_{0}=3 /\left(8 M^{3}\right)$. We have set $L=M$.

toward the origin). For $r$ close to $r_{\mathrm{h}}$ we can, to a good approximation, keep only the first two terms in the near horizon expansion,

$$
f(r) \approx 4 \pi T\left(r-r_{\mathrm{h}}\right)+a_{2}\left(r-r_{\mathrm{h}}\right)^{2} .
$$

We write $r=r_{\mathrm{h}}+\epsilon M$ where $\epsilon$ is a small quantity that we take to be $\pm 10^{-5}$. The plus sign is chosen for constructing the exterior solution, while the minus sign is chosen to construct the interior solution.

Of course, a priori $a_{2}$ is a free parameter in the near horizon solution. However, not all choices of $a_{2}$ will lead to a sensible solution at large distances. In fact, we find that $a_{2}$ must take on a uniquely determined value to join the numerical solution smoothly to the asymptotic expansion and avoid exciting the exponentially growing mode in (16) - we refer to this value as $a_{2}^{\star}$. The shooting method is used to determine this special value of $a_{2}^{\star}$, using the following idea. For a given choice of $M$ and the coupling $\mu$, a value $r_{\max }$ is chosen by the requirement that the asymptotic solution is a good approximation for $r>r_{\max }$. Then for a choice of $a_{2}$ the field equation is solved numerically, giving a value $f_{\text {numeric }}\left(r_{\text {max }} ; a_{2}\right)$ that can be compared with the asymptotic expansion. The scheme is successful when the numerically determined result $f_{\text {numeric }}\left(r_{\max } ; a_{2}\right)$ agrees with the asymptotic expansion at $r_{\max }$ - the result for $a_{2}^{\star}$ is shown in the left plot of Figure $1 .{ }^{5}$ By scanning the parameter space (see e.g., $[38,73]$ ) it can be confirmed that there is only one value of $a_{2}^{\star}$ for which this happens. In practice, $a_{2}^{\star}$ must be computed with very high precision in order to numerically solve the field equations at large distances. Inevitably, for any chosen precision, the numerical method will fail for large enough $r$, though this point of failure can be pushed to larger distances by increasing the precision of $a_{2}^{\star}$. The idea then is to use the numerical scheme to compute the solution up to $r_{\max }$, and then use the asymptotic solution to continue it toward infinity.

Once the value of $a_{2}^{\star}$ is determined through the procedure just described, the solution for $r<r_{\mathrm{h}}$ can be constructed. In this case the idea is to choose $\epsilon$ to be some small negative number to

\footnotetext{
${ }^{5}$ An additional step that one can perform is to compute the integrated residual $\int_{r_{\max }-\delta}^{r_{\max }}\left|f_{\text {numeric }}(r)-f_{\text {asymp }}(r)\right| d r$ where $\delta$ is some small positive quantity, and determine $a_{2}^{\star}$ through its minimization. This eliminates the possiblity that perhaps the numerical solution simply passes through the asymptotic solution at $r_{\max }$.
} 

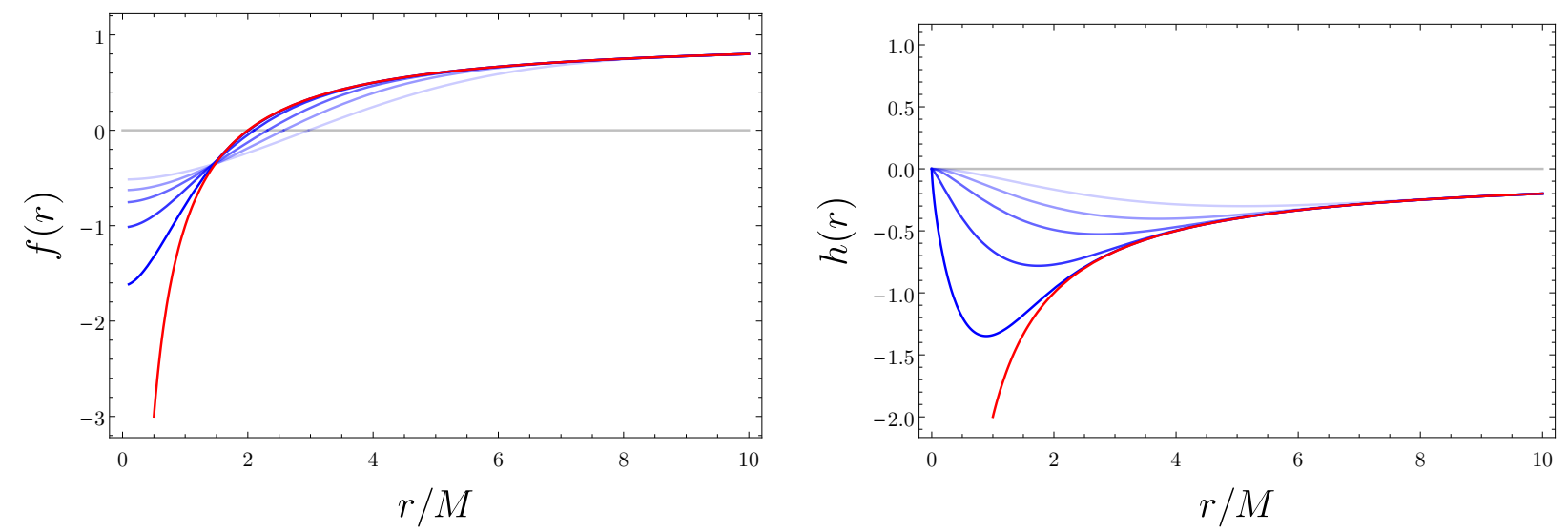

Figure 2: Here we show numerical profiles for the metric functions $f(r)$ (left) and $h(r) \equiv r^{2} p(r)$ (right). In each case, the blue curves correspond to $\mu=0.1,1,5,15,50$ in order of decreasing opacity, while the red curves illustrate the profile in Einstein gravity, given by $f(r)=1-2 M / r$ and $h(r)=-2 M / r$.

construct initial data just inside the horizon. The solution can then be constructed numerically for all $r<r_{\mathrm{h}}$, and no issues arise.

The basic idea for constructing the numerical solution for $g(r)$ is the same as for $f(r)$. Since the equation for $g(r)$ depends on $f(r)$ we first construct a numerical solution for $f(r)$ as just described. We then use the near horizon expansion for $g(r)$ to establish initial data for the numerical routine. The near horizon solution for $g(r)$ does not fix the parameter $g_{0}$, and so we once again have a oneparameter family of initial data. However, just as was the case for $a_{2}$ in the solution for $f(r)$, we find that $g_{0}$ must take on a uniquely fixed value $g_{0}^{\star}$ so that the numerical solution connects smoothly with the asymptotic expansion for $g(r)$, and avoids exciting the growing mode. The specific value for $g_{0}^{\star}$ is determined in a way completely analogous to the value of $a_{2}^{\star}$ : using the shooting method, terminating the procedure when the numerically constructed solution $g_{\text {numeric }}\left(r ; g_{0}\right)$ agrees with the asymptotic solution at a large value of $r$ where the asymptotic solution is a good approximation the result is shown in the right plot of Figure 1.

Once the solution for $g(r)$ is constructed, it can then be numerically integrated to determine $p(r)$. We start the numerical integration at $r=\infty$ so that $p(r) \rightarrow 0$ as $r \rightarrow \infty$, ensuring that the solution is written in a frame that does not rotate at infinity. In Figure 2 we show the numerically constructed profiles for the metric functions for different choices of the coupling. On the left we show $f(r)$, where we see that the effect of the higher-curvature corrections is to push the horizon outward and ensure that $f(r) \rightarrow$ constant as $r \rightarrow 0$. On the right we show $h(r)=r^{2} p(r)$, which is the combination appearing in $g_{t \phi}$, neglecting the spin parameter $a$ and the angular piece $\left(1-x^{2}\right)$. Here the curves all approach $h(r) \rightarrow 0$ as $r \rightarrow 0$, rather than decaying as $r^{-1}$ as is the case in Einstein gravity.

The fact that $h(r)$ is bounded in all its domain has some interesting consequences. For the Kerr solution, the slowly rotating approximation only works for large enough $r$. If $\chi=a / M \ll 1$ this approximation is valid up to the level of the horizon, but it always breaks down when $r \rightarrow 0$, because $g_{t \phi}$ diverges and rotation becomes important. However, in the case of ECG we can see that $g_{t \phi}=a h(r) \sin ^{2} \theta$ is bounded for all values of $r$ (more importantly, $g_{t \phi} / g_{\phi \phi}$ is bounded). This 

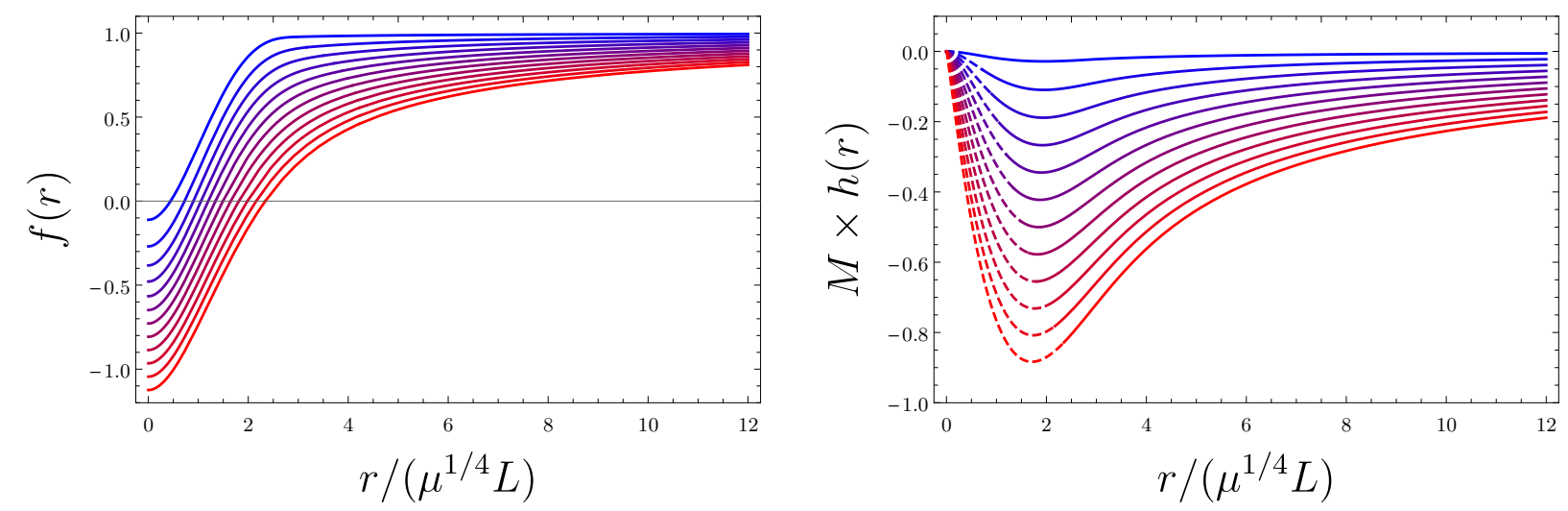

Figure 3: Numerical profiles for the metric functions $f(r)$ (left) and $M h(r)=M r^{2} p(r)$ (right) for fixed $\mu L^{4}$ and several values of the mass. From red to blue curves the mass ranges from $\frac{34}{30} \mu^{1 / 4} L$ to $\frac{1}{30} \mu^{1 / 4} L$. For fixed $\chi=a / M$, rotation becomes irrelevant when $M \ll \mu^{1 / 4} L$, and the zero-mass limit seems to correspond to a non-rotating extremal black hole of vanishing area. The dashed lines in the right plot correspond to the part of the solution which is inside the horizon.

means that if $\chi$ is small enough so that the maximum value of $g_{t \phi} / g_{\phi \phi}$ is also sufficiently small, the slowly rotating approximation could be valid for all $r$. More interestingly, we observe that when $M \rightarrow 0$ and we keep $\chi$ constant, the quantity $a h(r)=\chi M h(r)$ goes to zero everywhere. This is telling us that, in the regime where $M \ll L \mu^{1 / 4}$, the slowly rotating approximation is probably valid even for large $\chi$, since the effect of rotation is almost negligible. This seems to indicate that the maximum value for the angular momentum in these black holes could be larger than in general relativity. This is, there would be black holes with $a>M$ (and even with $a \gg M$ if $M$ is small). But in order to check this one would need at least to compute the solution at order $\mathcal{O}\left(a^{2}\right)$, to ensure that these terms are indeed irrelevant when $M \ll L \mu^{1 / 4}$.

In Fig. 2 we have plotted $f(r)$ and $h(r)$ for a fixed value of the mass and various values of the coupling $\mu L^{4}$, in order to explore how the solution is affected by the ECG correction. However, in practice we would have a fixed value of $\mu L^{4}$ and black holes of several masses. The effects of the corrections outside of the horizon only become relevant when the mass (or the horizon radius) is of the order of $\mu^{1 / 4} L$, and it is interesting to see how the profile of the solution changes with the mass. In Fig. 3 we show the profiles of $f(r)$ and $M h(r)$ for fixed $\mu L^{4}$ and various masses. As we mentioned, $M h(r)$ goes to 0 everywhere for small masses. We also observe a very interesting phenomenon in the case of $f(r)$ when $M \rightarrow 0$. While the radius $r_{\mathrm{h}}$ vanishes in that limit, the solution develops a potential well of length $\sim \mu^{1 / 4} L$ that is present for arbitrarily small mass. Thus, the zero mass limit of these black holes is not flat space. Instead, it seems to a be a massless, non-rotating extremal black hole whose horizon is reduced to a point (and coincides with a curvature singularity). This intriguing behavior will prove to have remarkable observational consequences.

\section{Properties of the solution}

In this section we study several physical properties of the solutions constructed above. Firstly, we evaluate the angular velocity of the horizon as a function of the mass. Then, we move on to 


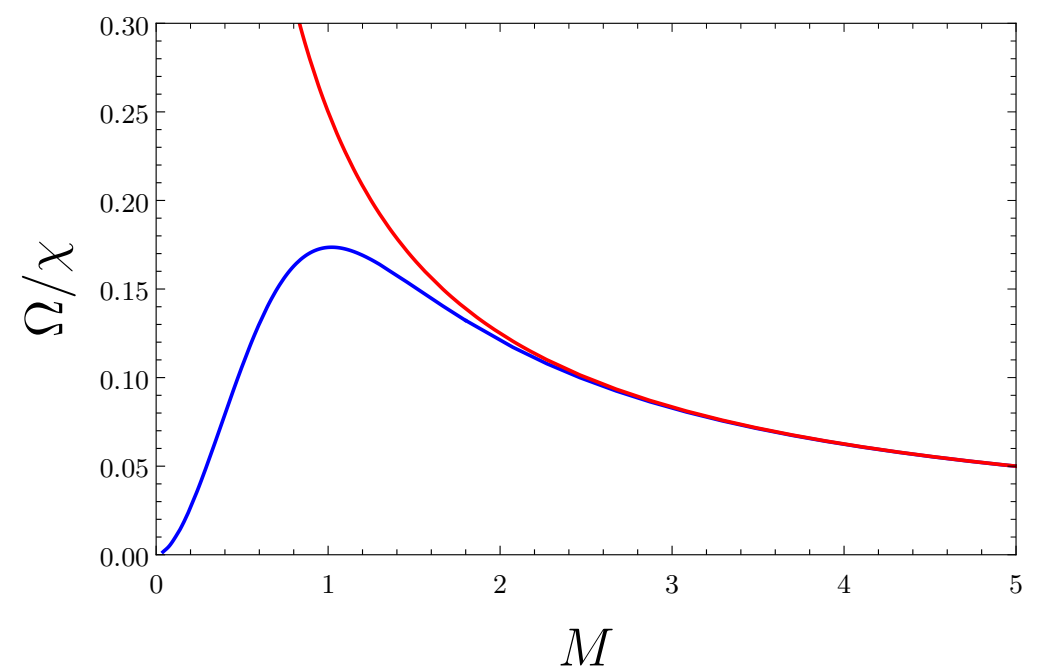

Figure 4: Angular velocity of the horizon as a function of the mass for fixed $\chi$. In red we show the result in Einstein gravity $(\Omega=\chi /(4 M))$ and in blue the case of ECG. The mass $M$ is expressed in units of $\mu^{1 / 4} L$ and $\Omega$ in the reciprocal units.

the study of geodesics. We find the general equations and then we compute the photon sphere. Then, restricting the discussion to geodesics in the equatorial plane, we compute the innermost stable circular orbit for timelike geodesics, photon rings and the Lyapunov exponents associated with their instability, and finally we study how the black hole shadow is modified with respect to the Einstein gravity case.

\subsection{Angular velocity of the horizon}

The angular velocity of the horizon is defined as

$$
\Omega=-\left.\frac{g_{t \phi}}{g_{\phi \phi}}\right|_{r=r_{\mathrm{h}}}
$$

In the perturbative regime, using Eqs. (11) and (12), we get

$$
\Omega=\frac{\chi}{4 M}\left[1-\frac{35 \mu L^{4}}{64 M^{4}}+\frac{26199 \mu^{2} L^{8}}{53248 M^{8}}+\ldots\right] .
$$

This expression works nicely for $M \gtrsim 1.7 \mu^{1 / 4} L$. For smaller values of $M$ we need to use the numerical solution, and in that case we get the profile of $\Omega$ shown in Fig. 4 . We observe that, unlike the Einstein gravity case, $\Omega$ no longer diverges for $M \rightarrow 0$. Instead, there is a maximum value $\Omega_{\max }$ that is reached at a mass $M\left(\Omega_{\max }\right)$ :

$$
\Omega_{\max } \approx \frac{0.1736 \chi}{\mu^{1 / 4} L}, \quad M\left(\Omega_{\max }\right) \approx 1.02 \mu^{1 / 4} L
$$

When $M \rightarrow 0$ the angular velocity also vanishes. Let us note that if we take $\chi$ to be small enough, the angular velocity remains small for every value of the mass, and hence the slowly spinning 
approximation should work for all masses. Nevertheless, for larger $\chi$ this approximation will break down at some point, and we expect the relation shown in Fig. 4 to be modified at small masses. An interesting question is whether a maximum value of $\Omega$ would persist in that case. In order to answer it we would need to find the solution at least at order $\mathcal{O}\left(a^{2}\right)$.

\subsection{Geodesics}

Let us study the geodesics of the slowly rotating solution. We can do that in general for a metric of the form

$$
d s^{2}=-f(r) d t^{2}+\frac{d r^{2}}{f(r)}+2 a h(r) \sin ^{2} \theta d t d \phi+r^{2}\left(d \theta^{2}+\sin ^{2} \theta d \phi^{2}\right) .
$$

The geodesics are most conveniently studied by introducing the Lagrangian

$$
L=\frac{1}{2} g_{a b} \dot{x}^{a} \dot{x}^{b},
$$

where $\dot{x}^{a}=d x^{a} / d \lambda$, and where $\lambda$ is an affine parameter. From the Lagrangian we may construct the conjugate momenta and the Hamiltonian, that read

$$
p_{a}=g_{a b} \dot{x}^{b}, \quad H=\frac{1}{2} g^{a b} p_{a} p_{b} .
$$

Then, we can write the Hamilton-Jacobi equation,

$$
\frac{\partial S}{\partial \lambda}=\frac{1}{2} g^{a b} \frac{\partial S}{\partial x^{a}} \frac{\partial S}{\partial x^{b}} .
$$

If we manage to solve this equation for the function $S$, then we get the solutions of the equations of motion using

$$
p_{a}=\frac{\partial S}{\partial x^{a}}
$$

We apply this method for the metric eq. (34). Since the Hamiltonian does not depend explicitly on $t, \phi$ and $\lambda$, we can write

$$
S=-\frac{1}{2} \xi^{2} \lambda-E t+\ell_{z} \phi+\tilde{S}(r, \theta),
$$

where $\xi^{2}, E$ and $\ell_{z}$ are constants. Let us further assume that the function $\tilde{S}(r, \theta)$ can be expressed

as $\tilde{S}(r, \theta)=\tilde{S}_{r}(r)+\tilde{S}_{\theta}(\theta)$. Inserting this into the Hamilton-Jacobi equation, we realize that it is indeed separable: ${ }^{6}$

$$
\left(\frac{\partial S_{\theta}}{\partial \theta}\right)^{2}+\frac{\ell_{z}^{2}}{\sin ^{2} \theta}=-r^{2} \xi^{2}+\frac{r^{2} E^{2}}{f(r)}+\frac{2 a h(r) E \ell_{z}}{f(r)}-r^{2} f(r)\left(\frac{\partial S_{r}}{\partial r}\right)^{2}
$$

Since the left-hand-side only depends on $\theta$ while the right-hand-side only depends on $r$, we conclude that both terms are equal to a constant, that we may call $j^{2}$. Therefore, we have determined all the derivatives of $S$,

$$
\frac{\partial S}{\partial t}=-E
$$

\footnotetext{
${ }^{6}$ We only keep terms linear in the spin $a$.
} 


$$
\begin{aligned}
\frac{\partial S}{\partial \phi} & =\ell_{z} . \\
\frac{\partial S}{\partial \theta} & = \pm \sqrt{j^{2}-\frac{\ell_{z}^{2}}{\sin ^{2} \theta}}, \\
\frac{\partial S}{\partial r} & = \pm \sqrt{-\frac{j^{2}}{r^{2} f(r)}-\frac{\xi^{2}}{f(r)}+\frac{E^{2}}{f(r)^{2}}+\frac{2 a h(r) E \ell_{z}}{r^{2} f(r)^{2}}} .
\end{aligned}
$$

Now we substitute $\partial_{a} S \rightarrow p_{a}$, where the momenta read

$$
p_{t}=-f(r) \dot{t}+a h(r) \sin ^{2} \theta \dot{\phi}, \quad p_{\phi}=a h(r) \sin ^{2} \theta \dot{t}+r^{2} \sin ^{2} \theta \dot{\phi}, \quad p_{\theta}=r^{2} \dot{\theta}, \quad p_{r}=\frac{\dot{r}}{f(r)}
$$

yielding a system of first-order equations,

$$
\begin{aligned}
r^{2} \dot{t} & =\frac{E r^{2}+a h(r) \ell_{z}}{f(r)}, \\
r^{2} \dot{\phi} & =\frac{\ell_{z}}{\sin ^{2} \theta}-\frac{a h(r) E}{f(r)} \\
r^{2} \dot{\theta} & = \pm \sqrt{j^{2}-\frac{\ell_{z}^{2}}{\sin ^{2} \theta}}, \\
\dot{r}^{2} & =-f(r)\left(\xi^{2}+\frac{j^{2}}{r^{2}}\right)+E^{2}+\frac{2 a h(r) E \ell_{z}}{r^{2}},
\end{aligned}
$$

where again we are expanding linearly in $a$. It is clear that, asymptotically, $j^{2}$ represents the total angular momentum of the orbit, while $\ell_{z}$ is the component of the angular momentum in the $z$ axis (this is, $\theta=0, \pi)$. On the other hand, $\xi^{2}$ is the norm of the tangent vector

$$
\xi^{2}=-g_{a b} \dot{x}^{a} \dot{x}^{b}
$$

\subsubsection{The photon sphere}

For null geodesics, we have $\xi^{2}=0$, and rescaling the affine parameter $\lambda$, we can always choose $E=1$. Let us then write the equation for the radial coordinate as

$$
\dot{r}^{2}+V_{\mathrm{ph}}(r)=0, \quad \text { where } \quad V_{\mathrm{ph}}(r)=\frac{j^{2} f(r)-2 a \ell_{z} h(r)}{r^{2}}-1 .
$$

The photon sphere is formed by constant- $r$ photon orbits, that appear when

$$
V_{\mathrm{ph}}\left(r_{\mathrm{ps}}\right)=0, \quad V_{\mathrm{ph}}^{\prime}\left(r_{\mathrm{ps}}\right)=0 .
$$

These conditions give us the radius of these orbits $r_{\mathrm{ps}}$ as well as the value of $j_{\mathrm{ps}}^{2}$. Since we are working perturbatively in $a$, let us write these quantities as

$$
r_{\mathrm{ps}}=r_{\mathrm{ps}}^{(0)}+a r_{\mathrm{ps}}^{(1)}, \quad j_{\mathrm{ps}}^{2}=\left(j_{\mathrm{ps}}^{(0)}\right)^{2}+a\left(j_{\mathrm{ps}}^{(1)}\right)^{2} .
$$

We find that $r_{\mathrm{ps}}^{(0)}$ is determined by the equation

$$
r_{\mathrm{ps}}^{(0)} f^{\prime}\left(r_{\mathrm{ps}}^{(0)}\right)-2 f\left(r_{\mathrm{ps}}^{(0)}\right)=0
$$


and the rest of quantities read

$$
\begin{aligned}
& r_{\mathrm{ps}}=r_{\mathrm{ps}}^{(0)}+\left.\frac{2 a \ell_{z} f\left(r h^{\prime}-2 h\right)}{r\left(r^{2} f^{\prime \prime}-2 f\right)}\right|_{r=r_{\mathrm{ps}}^{(0)}}, \\
& j_{\mathrm{ps}}^{2}=\frac{\left(r_{\mathrm{ps}}^{(0)}\right)^{2}}{f\left(r_{\mathrm{ps}}^{(0)}\right)}+\frac{2 a \ell_{z} h\left(r_{\mathrm{ps}}^{(0)}\right)}{f\left(r_{\mathrm{ps}}^{(0)}\right)}
\end{aligned}
$$

Thus, every choice of $\ell_{z}$ produces a family of constant- $r$ geodesics. Observe that in the case of equatorial orbits we have $\ell_{z}= \pm\left|j_{\mathrm{ps}}\right|$ and this fixes two types of orbits, either prograde or retrograde.

In the case at hand, when $\mu L^{4} / M^{4} \ll 1$ we may use the perturbative solution and we get

$$
\begin{aligned}
& r_{\mathrm{ps}}=3 M+\frac{13 \mu L^{4}}{81 M^{3}}+\frac{1295 \mu^{2} L^{8}}{59049 M^{7}}-\frac{a \ell_{z}}{M}\left[\frac{2}{9}-\frac{460 \mu L^{4}}{6561 M^{4}}+\frac{764 \mu^{2} L^{8}}{1594323 M^{8}}\right] . \\
& j_{\mathrm{ps}}^{2}=27 M^{2}+\frac{35 L^{4} \mu}{27 M^{2}}-\frac{2806 L^{8} \mu^{2}}{19683 M^{6}}+\frac{a \ell_{z}}{M}\left[4 M-\frac{140 L^{4} \mu}{243 M^{3}}-\frac{48790 L^{8} \mu^{2}}{2302911 M^{7}}\right] .
\end{aligned}
$$

On the other hand, when $\mu L^{4} / M^{4}$ becomes of order 1 or larger we need to use the numerical solution — see section 3.4 below.

\subsection{Geodesics in the equatorial plane}

Here we consider geodesics confined to the equatorial plane, i.e., $x=0$. For this purpose, we will specialize the results from the previous subsection to this situation, which amounts to setting $\theta=\pi / 2$ and $\dot{\theta}=0$. Note that, via these two constraints it is enforced that $j^{2}=\ell_{z}^{2}$. We can understand eq. (49) as analogous to that describing a particle moving in a potential,

$$
\dot{r}^{2}+V_{\text {eff }}(r)=0, \quad \text { where } \quad V_{\text {eff }}(r) \equiv f(r)\left(\mu^{2}+\frac{j^{2}}{r^{2}}\right)-\frac{2 a h(r) E j}{r^{2}}-E^{2} .
$$

In the following two sub-sections, we will consider the special case of circular orbits, determining the inner-most stable circular orbit (ISCO) for timelike geodesics, and the photon ring for null geodesics.

\subsubsection{Timelike geodesics: ISCO}

First let us consider the case of circular, timelike geodesics, i.e., those with $\xi^{2}=1$ and $\dot{r}=0$. The conditions for the existence of these geodesics are

$$
V_{\text {eff }}(r)=0, \quad V_{\text {eff }}^{\prime}(r)=0,
$$

and the stability of the circular orbit can be deduced by considering the sign of $V_{\text {eff }}^{\prime \prime}(r)$, with $V_{\text {eff }}^{\prime \prime}(r)>0$ indicating stability and $V_{\text {eff }}^{\prime \prime}(r)<0$ indicating instability. We can determine the location of the inner-most stable circular orbit by searching for circular orbits that are also inflection points, i.e., orbits for which $V_{\mathrm{eff}}^{\prime \prime}(r)=0$, leading to three conditions on the parameters $r, E$, and $j$. 
We wish to solve these equations, working to linear order in the rotation parameter. To this end, we make the following definitions:

$$
r_{\mathrm{ISCO}}=r_{\mathrm{ISCO}}^{(0)}+a r_{\mathrm{ISCO}}^{(1)}, \quad E_{\mathrm{ISCO}}=E_{\mathrm{ISCO}}^{(0)}+a E_{\mathrm{ISCO}}^{(1)}, \quad j_{\mathrm{ISCO}}=j_{\mathrm{ISCO}}^{(0)}+a j_{\mathrm{ISCO}}^{(1)} .
$$

Substituting these into the conditions $d^{n} V_{\text {eff }}(r) / d r^{n}=0$ for $n=0,1,2$ and expanding to linear order in $a$ yields a system of six equations that must be solved. These equations themselves are not particularly illuminating, but let us note that within the perturbative regime they admit the following solution:

$$
\begin{aligned}
& r_{\mathrm{ISCO}}=6 M\left[1+\frac{101 \mu L^{4}}{3888 M^{4}}-\frac{499601 \mu^{2} L^{8}}{362797056 M^{8}}\right] \mp 4 a \sqrt{\frac{2}{3}}\left[1-\frac{10073 \mu L^{4}}{93312 M^{4}}+\frac{92651747 \mu^{2} L^{8}}{17414258688 M^{8}}\right], \\
& E_{\mathrm{ISCO}}=\frac{2 \sqrt{2}}{3}\left[1+\frac{267 \mu L^{4}}{279936 M^{4}}-\frac{782985 \mu^{2} L^{8}}{17414258688 M^{8}}\right] \mp \frac{a}{18 \sqrt{3} M}\left[1-\frac{499 \mu L^{4}}{5184 M^{4}}+\frac{20883769 \mu^{2} L^{8}}{2358180864 M^{8}}\right], \\
& j_{\mathrm{ISCO}}= \pm 2 \sqrt{3} M\left[1+\frac{59 \mu L^{4}}{11664 M^{4}}-\frac{355163 \mu^{2} L^{8}}{2176782336 M^{8}}\right]-\frac{2 \sqrt{2} a}{3}\left[1+\frac{4603 \mu L^{4}}{93312 M^{4}}-\frac{21098789 \mu^{2} L^{8}}{5804752896 M^{8}}\right] .
\end{aligned}
$$

Here taking the upper sign describes the ISCO for prograde orbits $\left(j_{\mathrm{ISCO}}^{(0)}>0\right)$, while the lower sign describes the ISCO for retrograde orbits $\left(j_{\text {ISCO }}^{(0)}<0\right)$. The perturbative solution provides an accurate description when $M /\left(\mu^{1 / 4} L\right) \gtrsim 1.5$, but for smaller masses we must resort to the numerical solution. The results for each of the parameters are plotted in Figure 5. The corrections due to ECG become most significant for small masses, where they can either increase or decrease the relevant parameters.

\subsubsection{Null geodesics: photon rings}

Let us now consider how the rotation deforms the photon rings of the black hole. The photon rings are circular orbits (i.e., having $r=$ constant) for null geodesics lying in the equatorial plane $x=0$. We therefore must seek determine the simultaneous zeros of the effective potential and its first derivative. In this case, rather than work with the conserved quantities $E$ and $j$, we will use the angular velocity $\omega \equiv d \phi / d t$, which is conserved along the photon trajectory. Written in terms of the angular velocity, the conditions determining the location of the photon rings read

$$
\begin{aligned}
& 0=\omega^{2} r^{2}+2 a \omega h(r)-f(r), \\
& 0=2 \omega^{2} r+2 a \omega h^{\prime}(r)-f^{\prime}(r) .
\end{aligned}
$$

To solve these equations to first order in $a$ it is useful to consider first the case where the rotation vanishes, corresponding to the photon sphere of the static, spherically symmetric solution. The equations reduce to

$$
\frac{r_{\mathrm{ps}} f^{\prime}\left(r_{\mathrm{ps}}\right)}{2}-f\left(r_{\mathrm{ps}}\right)=0, \quad \omega_{\mathrm{ps}}=\frac{\sqrt{f\left(r_{\mathrm{ps}}\right)}}{r_{\mathrm{ps}}},
$$

where the first equation determines $r_{\mathrm{ps}}$ - the radius of the photon sphere in the static solution - and we find that the solution is unique. Once $r_{\mathrm{ps}}$ is known, the second equation determines the angular velocity. When the rotation is non-trivial, Eqs. (63) and (64) admit two distinct solutions 

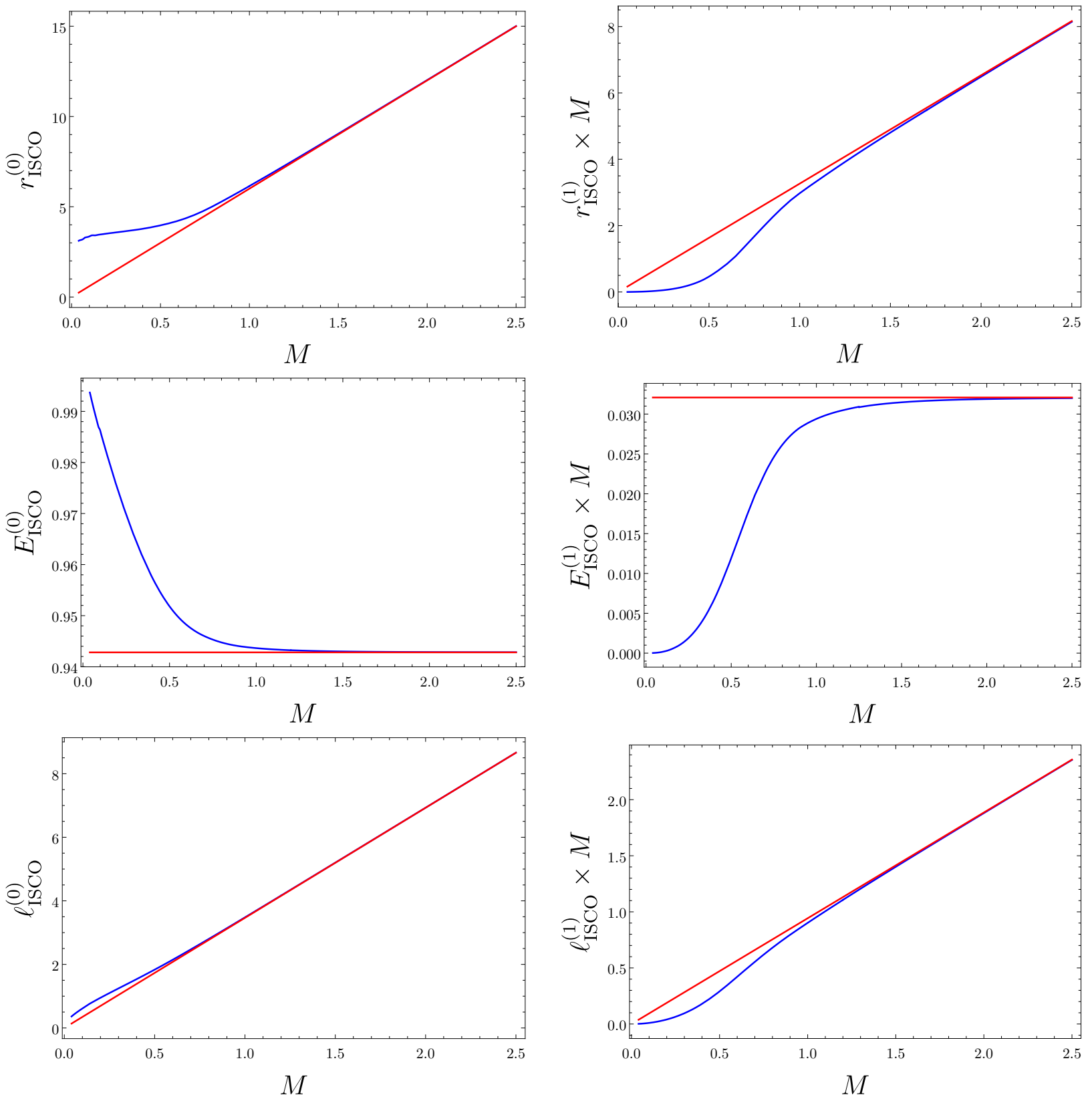

Figure 5: Here we plot the parameters describing the ISCO. In the left column we present the zeroth-order terms, which are those corresponding to the static solution. The right column shows the leading-order correction due to rotation. In each case, the blue curves represent the ECG result, while the red curves represent the Einstein gravity result. In all cases the mass is expressed in units of $\mu^{1 / 4} L$. 
for the radius of the photon rings and the angular velocity. We write the corrected radius of the photon ring, denoted $r_{\mathrm{pr}_{ \pm}}$, in the following way

$$
r_{\mathrm{pr}_{ \pm}}=r_{\mathrm{ps}} \pm a r_{\mathrm{pr}}^{(1)}
$$

where the leading order correction $r_{\mathrm{pr}}^{(1)}$ is given by

$$
r_{\mathrm{pr}}^{(1)}=\frac{2 \sqrt{f\left(r_{\mathrm{ps}}\right)}\left[r_{\mathrm{ps}} h^{\prime}\left(r_{\mathrm{ps}}\right)-2 h\left(r_{\mathrm{ps}}\right)\right]}{2 f\left(r_{\mathrm{ps}}\right)-r_{\mathrm{ps}}^{2} f^{\prime \prime}\left(r_{\mathrm{ps}}\right)},
$$

and so it can be directly computed from the numeric/approximate solutions once the value of $r_{\mathrm{ps}}$ characterizing the static solution is known. The results can then be plugged into the expression for the angular velocity and expanding to linear order in $a$, we obtain two solutions

$$
\omega_{\mathrm{pr} \pm}=\mp \omega_{\mathrm{ps}}^{(0)}+a \omega_{\mathrm{pr}}^{(1)}=\mp \frac{\sqrt{f\left(r_{\mathrm{ps}}\right)}}{r_{\mathrm{ps}}}-a \frac{h\left(r_{\mathrm{ps}}\right)}{r_{\mathrm{ps}}^{2}} .
$$

where the plus sign corresponds to the prograde photon ring and the minus sign corresponds to the retrograde photon ring.

Using the perturbative expansions for the metric functions, we can write

$$
\begin{aligned}
& r_{\mathrm{pr}}=3 M+\frac{13 \mu L^{4}}{81 M^{3}}+\frac{1295 \mu^{2} L^{8}}{59049 M^{7}} \pm a\left(\frac{2}{\sqrt{3}}-\frac{425 \mu L^{4}}{729 \sqrt{3} M^{4}}-\frac{13033 \mu^{2} L^{8}}{2125764 \sqrt{3} M^{8}}\right), \\
& \omega_{\mathrm{pr}}=\mp\left(\frac{1}{3 \sqrt{3} M}-\frac{35 \mu L^{4}}{4374 \sqrt{3} M^{5}}-\frac{7549 \mu^{2} L^{8}}{12754584 \sqrt{3} M^{9}}\right)+a\left(\frac{2}{27 M^{2}}-\frac{280 \mu L^{4}}{19683 M^{6}}-\frac{6247 \mu^{2} L^{8}}{62178597 M^{10}}\right)
\end{aligned}
$$

In Figure 6 we plot the corrections to the radius and angular velocity of the photon sphere as a function of the black hole mass. In the case of the zeroth-order terms, which correspond to the static solution, the ECG corrections are most prominent at small mass.

In Figure 7 we depict the ratio $\omega_{+} / \mid \omega_{-}$for several values of the higher-order coupling. The idea here is the same as that in [69] — in Einstein gravity this ratio is controlled only by the spin parameter, while here it depends also on the higher-order coupling. This feature could, in principle, be used to constrain the values of the ECG coupling, provided the spin parameter could be independently measured. From this plot we note that the effect of the ECG correction is to push this ratio below the corresponding curve for Einstein gravity. As the ECG coupling increases, the curves begin to "bunch up" - in other words, the ratio is most sensitive to small differences in the coupling when the coupling is small.

We turn next to the question of stability of the photon ring orbits. Of course, it comes as no surprise that these orbits are unstable. Our goal is to compute the Lyapunov exponent associated with this instability. It has long been known [75] that the quasi-normal mode frequencies, in the Eikonal approximation, are related to the properties of unstable null geodesics. Specifically, the real part of the quasi-normal mode frequencies is related to the angular velocity of the unstable null orbit, while the imaginary part is related to the Lyapunov exponent. For any static, spherically symmetric spacetime one can prove this relationship to be [76]:

$$
\omega_{\mathrm{QNM}}=\omega_{\mathrm{ps}} \ell-i(n+1 / 2)|\lambda|
$$



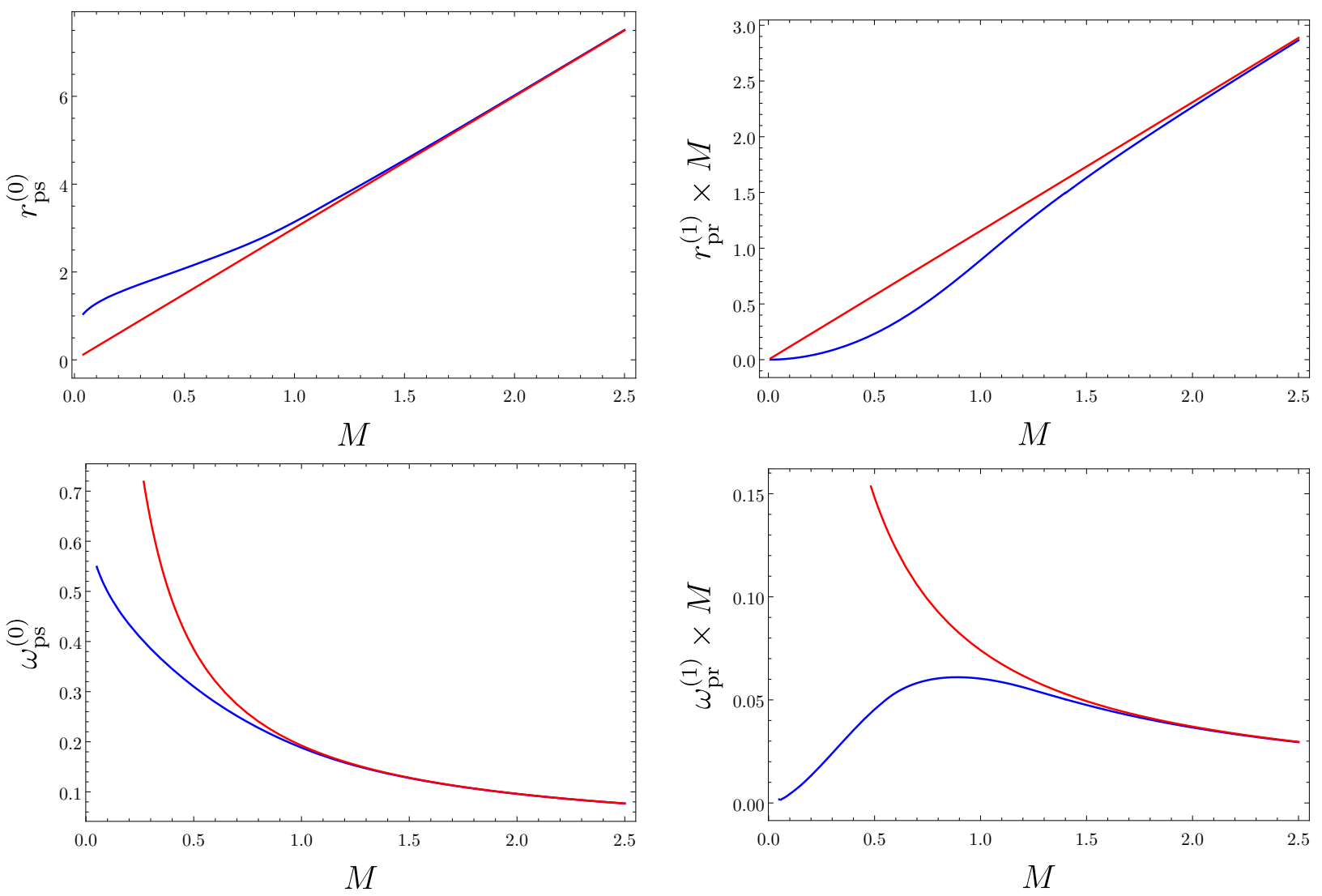

Figure 6: Parameters describing the photon ring. The top row shows the radius of the photon ring, while the bottom row shows the angular velocity of the photon ring. The left column shows these parameters in the static case, while the right column shows the leading-order corrections due to rotation. In each plot the mass is expressed in units of $\mu^{1 / 4} L$, and the red curves indicate the Einstein gravity result.

where $\lambda$ is the Lyapunov exponent associated with the orbit.

To extract the Lyapunov exponent, we will follow [75]. We consider a solution of the equatorial geodesic equations corresponding to the photon ring. We then perturb those equations according to

$$
r(t)=r_{\mathrm{ps}}(1+\epsilon F(t)), \quad s(t)=\frac{t}{\alpha}+\epsilon G(t), \quad \phi(t)=\left|\omega_{ \pm}\right|(1+\epsilon H(t))
$$

where $\epsilon$ is a small parameter controlling the perturbation and the system is subject to the boundary condition that the perturbation vanishes at $t=0$. In the above, the constant $\alpha$ gives the relationship between the affine parameter $s$ and the coordinate time $t$ in the absence of the perturbation:

$$
\left.\alpha \equiv \dot{t}\right|_{r \rightarrow r_{\mathrm{ps}}} .
$$

The only relevant correction for our purposes here is $F(t)$. This can be obtained via expanding the equation (59) involving the effective potential to first-order in the perturbation. The differential equation reads

$$
\frac{d^{2} r}{d s^{2}}+\frac{1}{2} \frac{d V_{\mathrm{eff}}}{d r}=0 .
$$




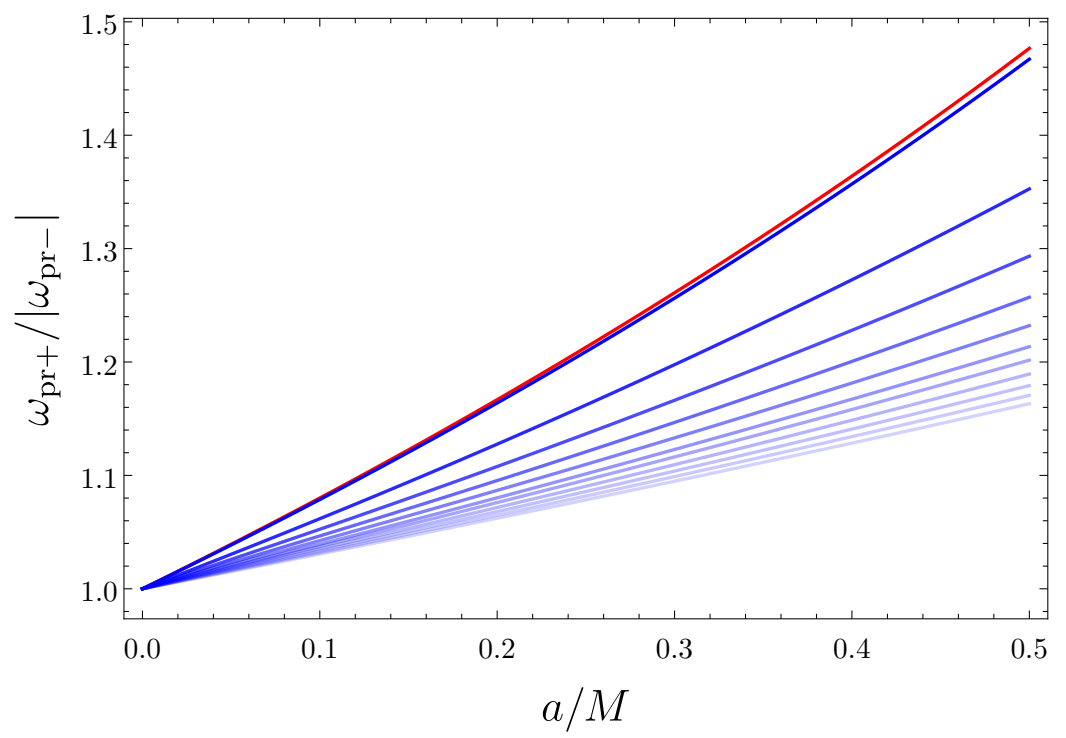

Figure 7: Here we show the ratio of angular velocities for the photon rings as a function of the spin parameter $a / M$. The red curve corresponds to Einstein gravity, while the blue curves correspond to different values of $\mu$ ranging from $\mu=1 / 10$ (darkest curve) to $\mu=74 / 5$ (lightest curve), with the intermediate curves spaced by $\Delta \mu=3 / 2$. We have set $L=M$.

When we expand this to leading order in $\epsilon$, we obtain

$$
2 \alpha^{2} F^{\prime \prime}(t)+V_{\mathrm{eff}}^{\prime \prime}\left(r_{\mathrm{ps}}\right) F(t)=0 .
$$

Imposing the condition $F(0)=0$ gives the solution

$$
F(t) \propto \sinh \lambda t
$$

where

$$
\lambda^{2} \equiv-\frac{V_{\mathrm{eff}}^{\prime \prime}\left(r_{\mathrm{ps}}\right)}{2 \alpha^{2}}
$$

As with the other quantities, we write the Lyapunov exponent as a zeroth-order term plus a correction linear in $a$ :

$$
\lambda=\lambda^{(0)}+a \lambda^{(1)} .
$$

Although expressions for $\lambda^{(0)}$ and $\lambda^{(1)}$ can easily be obtained in terms of the metric functions, the resulting expressions are quite messy. When the mass is large we can obtain a perturbative solution for the Lyapunov exponent

$$
\lambda=\frac{1}{2 \sqrt{3} M}+\frac{91 \mu L^{4}}{4374 \sqrt{3} M^{5}}+\frac{50987 \mu^{2} L^{8}}{12754584 \sqrt{3} M^{9}} \mp a\left(\frac{28 \mu L^{4}}{729 M^{6}}-\frac{1531 \mu^{2} L^{8}}{177147 M^{10}}\right)
$$

but when the mass, expressed in units of $\mu^{1 / 4} L$, becomes small we must, as before, resort to numerics. The results are shown in Figure 8. The zeroth-order term, which corresponds to the Lyapunov exponent for the static solutions, differs significantly from the Einstein gravity result at small mass. In particular, it reaches a maximum before turning rapidly toward zero in the small mass regime. This behavior is somewhat similar to that seen for rotating black holes in the extremal 

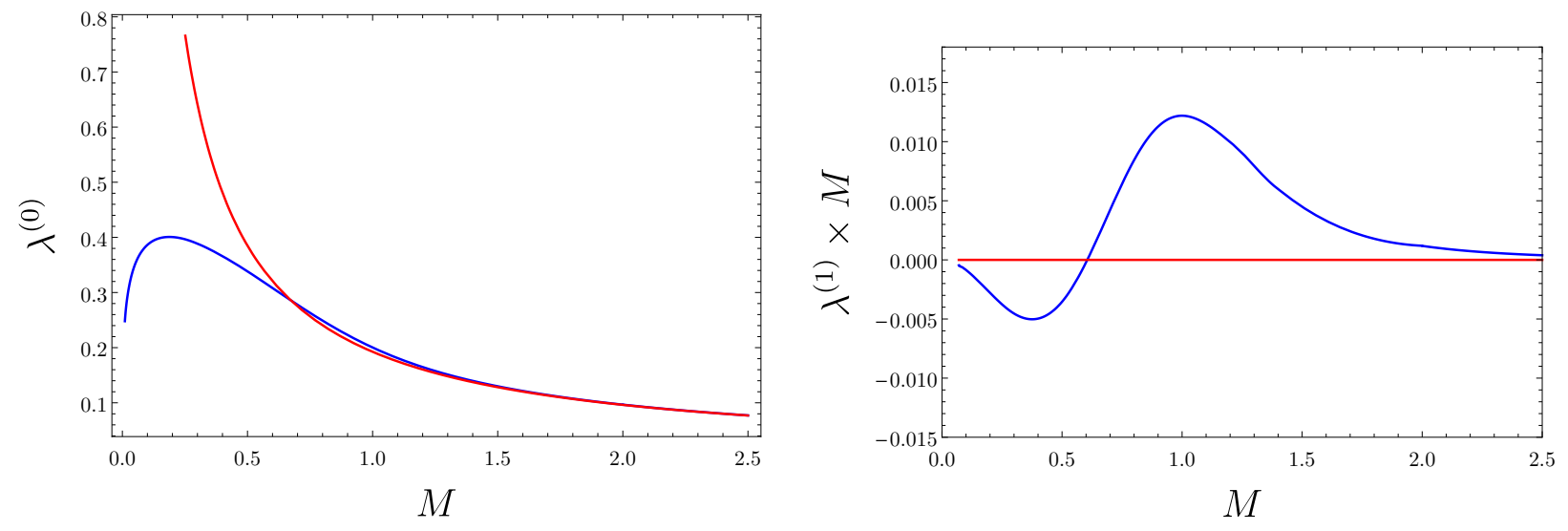

Figure 8: Lyapunov exponent for photon ring. The left panel shows the zeroth-order term, corresponding to the static solution, while the right panel shows the leading correction due to rotation. In each case the red curve corresponds to the Einstein gravity result, while the blue curve is the ECG case. The mass is expressed in units of $\mu^{1 / 4} L$.

limit [76]. The leading-order correction due to rotation oscillates around the Einstein gravity value, with the ECG corrections becoming negligible at both small and large masses. The effect of this oscillation is to introduce a mass dependence to the slope of the Lynapunov exponent (as a function of $a$ ) in ECG.

\subsection{Black hole shadow}

Let us consider an observer that is placed far from the black hole, at a radius $r_{0}$, polar angle $\theta_{0}$ and, without loss of generality $\phi_{0}=0$. Then this observer receives a photon that moves in the direction $d r / d t>0$ and whose trajectory is defined by the angular momentum parameters $j^{2}$ and $\ell_{z}$. We want to determine the angle of incidence of this photon to the plane perpendicular to the $r$ direction at the position of the observer. The spatial tangent vector at that point is

$$
u=-\dot{r} e_{r}+r_{0} \dot{\theta} e_{\theta}+r_{0} \sin \theta_{0} \dot{\phi} e_{\phi},
$$

where we have introduced the following orthonormal system for the observer, who is looking directly toward the black hole

$$
e_{r}=-\partial_{r}, \quad e_{\theta}=\frac{\partial_{\theta}}{r_{0}}, \quad e_{\phi}=\frac{\partial_{\phi}}{r_{0} \sin \theta_{0}} .
$$

Let us define $\pi / 2-\delta$ as the angle of incidence of the photon on the plane $r=r_{0}$ and $\alpha$ as the angle that the projected vector forms with the direction $e_{\phi}$. In other words, we parametrize the tangent vector as

$$
\begin{gathered}
u=-\dot{r} e_{r}+\sin \delta\left(e_{\theta} \sin \alpha+e_{\phi} \cos \alpha\right) \\
\sin \delta=r_{0} \sqrt{\dot{\theta}^{2}+\sin ^{2} \theta_{0} \dot{\phi}^{2}}, \quad \cos \alpha=\frac{\sin \theta_{0} \dot{\phi}}{\sqrt{\dot{\theta}^{2}+\sin ^{2} \theta_{0} \dot{\phi}^{2}}}
\end{gathered}
$$

In these expressions we have already assumed that $r_{0} \sqrt{\dot{\theta}^{2}+\sin ^{2} \theta_{0} \dot{\phi}^{2}} \ll 1$ because we are taking $r_{0} \rightarrow \infty$. Finally, using the geodesic equations, we can write these angles in terms of the angular 

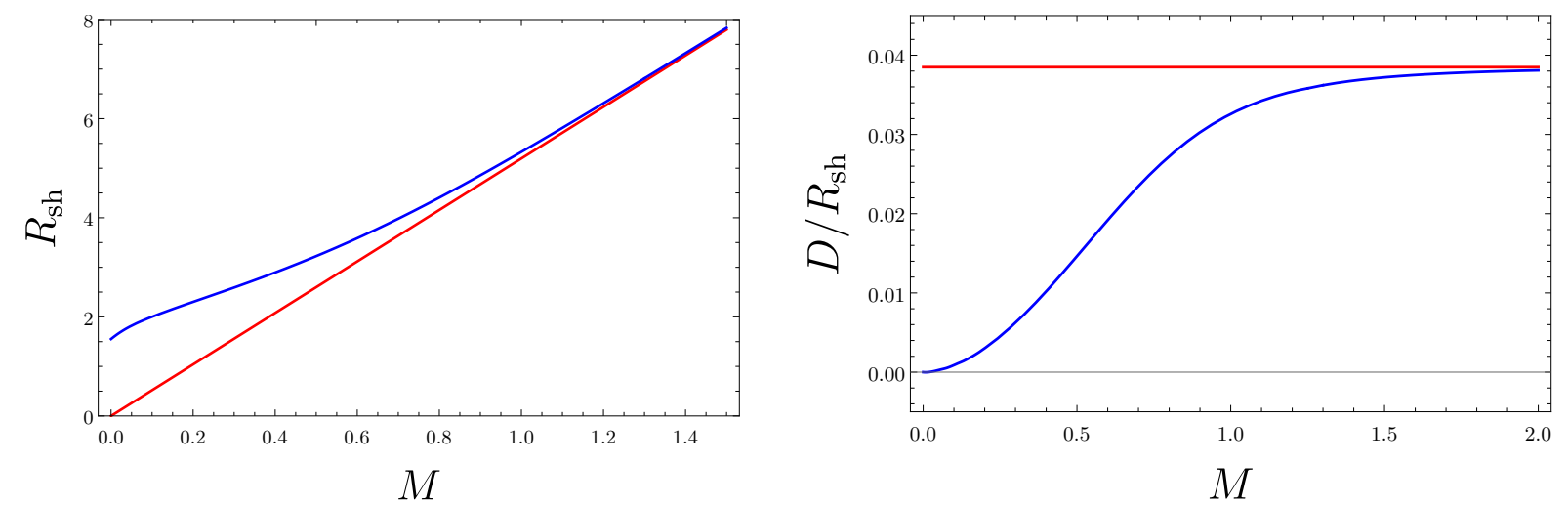

Figure 9: Properties of the black hole shadow for Einstein gravity (red curve) and Einsteinian cubic gravity (blue curve). Left: we show the radius $R_{\text {sh }}$ of the shadow as a function of the mass $M$. Right: we plot the ratio between the shift of the shadow $D$ and the radius $R_{\mathrm{sh}}$ as a function of the mass for $\chi=-0.1$. The mass is expressed in units of $\mu^{1 / 4} L / G$ and $R_{\mathrm{sh}}$ in the reciprocal length units.

momentum; or conversely, we can express the angular momentum of the geodesic in terms of the angles:

$$
j=r_{0} \sin \delta, \quad \ell_{z}=r_{0} \sin \theta_{0} \cos \alpha \sin \delta .
$$

Now, the shadow of the black hole is determined by the photons that pass arbitrarily close to the photon sphere. Remember that for the photons in the photon sphere we have derived a relation between $j^{2}$ and $\ell_{z}$, and therefore the contour of the shadow corresponds precisely to photons with that value of the angular momentum. Taking into account these points, we derive the following equation

$$
r_{0}^{2} \sin ^{2} \delta=\frac{\left(r_{\mathrm{ps}}^{(0)}\right)^{2}}{f\left(r_{\mathrm{ps}}^{(0)}\right)}+\frac{2 a \sin \theta_{0} h\left(r_{\mathrm{ps}}^{(0)}\right)}{f\left(r_{\mathrm{ps}}^{(0)}\right)} \cos \alpha r_{0} \sin \delta,
$$

that determines the contour $\delta(\alpha)$ of the black hole shadow. Expanding linearly in $a$, the solution to this equation reads

$$
r_{0} \sin \delta=\frac{r_{\mathrm{ps}}^{(0)}}{\sqrt{f\left(r_{\mathrm{ps}}^{(0)}\right)}}+\frac{a \sin \theta_{0} h\left(r_{\mathrm{ps}}^{(0)}\right)}{f\left(r_{\mathrm{ps}}^{(0)}\right)} \cos \alpha,
$$

Since $\delta \ll 1$ when $r_{0}>r_{\mathrm{ps}}^{(0)}$, we can approximate $\sin \delta \approx \delta$, and we can see that the curve $\delta(\alpha)$ is approximately a circumference of radius $R_{\mathrm{sh}}$ centered at $\alpha=0, r_{0} \delta=D$, where

$$
R_{\mathrm{sh}}=\frac{r_{\mathrm{ps}}^{(0)}}{\sqrt{f\left(r_{\mathrm{ps}}^{(0)}\right)}}, \quad D=-\frac{a \sin \theta_{0} h\left(r_{\mathrm{ps}}^{(0)}\right)}{f\left(r_{\mathrm{ps}}^{(0)}\right)} .
$$

Thus, at first order in $a$, the effect of rotation is to shift the shadow a distance $D$ from the radial direction. Let us compute $R_{\text {sh }}$ and $D$ for the slowly rotating ECG black hole. First, if $\mu L^{4} / M^{4} \ll 1$ we can perform a perturbative analysis and we find

$$
R_{\mathrm{sh}}=3 \sqrt{3} M+\frac{35 L^{4} \mu}{162 \sqrt{3} M^{3}}+\frac{1111 L^{8} \mu^{2}}{52488 \sqrt{3} M^{7}}, \quad D=-\frac{a \sin \theta_{0}}{M}\left(2 M-\frac{70 L^{4} \mu}{243 M^{3}}-\frac{24395 L^{8} \mu^{2}}{2302911 M^{7}}\right) .
$$




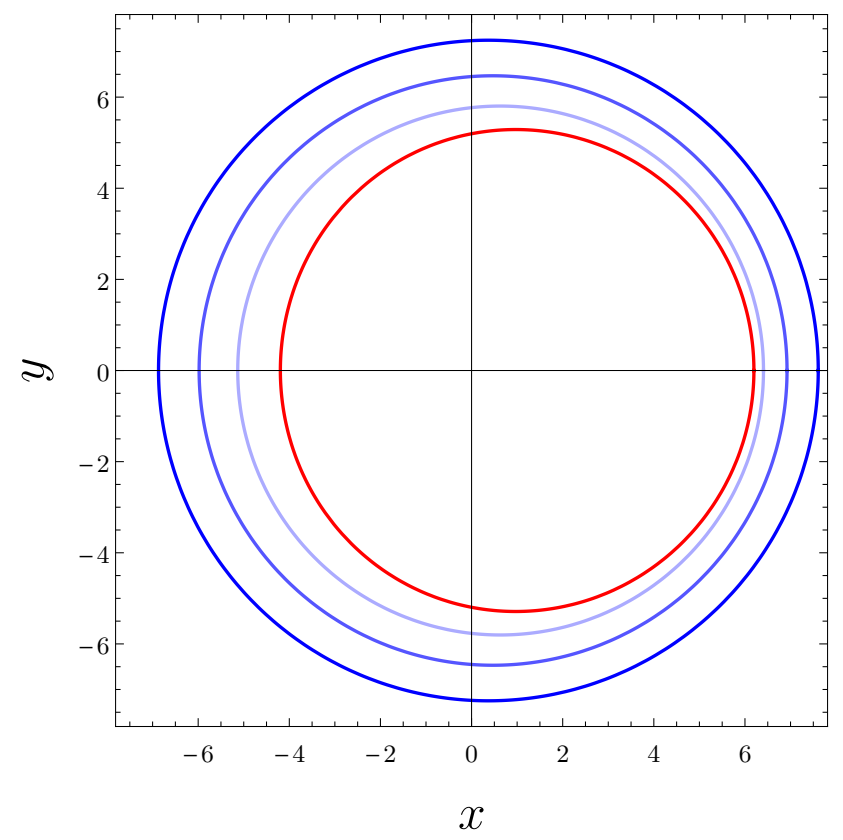

Figure 10: Contour of the black hole shadow for a fixed mass and for $\chi=-0.5$. In red we show the Einstein gravity result and in blue the ECG one for several values of the higher-order coupling. From lighter to darker blue: $\mu^{1 / 4} L / M=1.5,2,2.5$.

For large $\mu L^{4} / M^{4}$ we need to use the numerical solution, and we find in that case some remarkable facts. First, we observe that when we decrease $M$ (keeping $a / M$ fixed), the ratio $D / M$ goes to zero, which is a manifestation of the fact that the effect of rotation becomes irrelevant for these black holes when the mass is small.

More strikingly, we find that when $M \rightarrow 0$, the radius of the shadow $R_{\mathrm{sh}}$ tends to a constant value. Performing a fit to the numerical values, we get that the behavior for $M \ll L \mu^{-1 / 4}$ is

$$
R_{\mathrm{sh}} \approx \frac{1.6 L}{\mu^{1 / 4}}+4.86 M \quad \text { when } M \rightarrow 0
$$

In Fig. 9 we show the profile of $R_{\mathrm{sh}}$ and $D / R_{\mathrm{sh}}$ as functions of the mass and in Fig. 10 we plot the contour of the shadow. We observe that the effect of the ECG term is to make the shadow larger and more centered with respect to the situation in Einstein gravity.

\section{Asymptotically AdS solutions}

In this section we study the case of slowly rotating black holes in ECG with a negative cosmological constant. The action reads

$$
I=\frac{1}{16 \pi G} \int d^{4} x \sqrt{-g}\left[\frac{6}{L^{2}}+R-\frac{\mu L^{4}}{8} \mathcal{P}\right]
$$


where now $L$ would coincide with the AdS scale if the corrections were not present. Instead, the AdS radius $\tilde{L}=L / \sqrt{f_{\infty}}$ is determined by the cubic equation

$$
h\left(f_{\infty}\right)=1-f_{\infty}+\mu f_{\infty}^{3}=0 .
$$

Here we have introduced the function $h(x)$ which we will refer to as the embedding function. ${ }^{7}$

The ansatz for the slowly rotating black holes takes the form,

$$
d s^{2}=-N(r)^{2} f(r) d t^{2}+\frac{d r^{2}}{f(r)}+2 a r^{2} p(r)\left(1-k x^{2}\right) d t d \phi+r^{2}\left[\frac{d x^{2}}{1-k x^{2}}+\left(1-k x^{2}\right) d \phi^{2}\right],
$$

where again we work to linear order in the rotation parameter $a$. Unlike the asymptotically flat case, we can also consider planar $k=0$ or hyperbolic $k=-1$ transverse sections, besides spherical ones $k=1$. We will mainly focus on the latter case, but we will write the basic equations for general $k$. The equations of motion can be reduced, again, to two second-order equations for $f$ and for $g=p^{\prime}$, with two integration constants, $M$ and $C$ :

$$
\begin{aligned}
2 G M & =r^{3} / L^{2}+r(k-f)+\frac{\mu L^{4}}{4 r^{2}}\left[6\left(\frac{r f^{\prime}}{2}+k-f\right) f^{\prime \prime} f-\left(r^{2} f^{\prime 2}+3 r k f^{\prime}+6 f(k-f)\right) f^{\prime}\right], \\
C & =r^{4} g-\frac{3 \mu L^{4}}{2}\left[\left(\frac{r f^{\prime}}{2}+k-f\right) r^{2} f g^{\prime \prime}+\left(\frac{r^{2} f}{2} f^{\prime \prime}+\frac{r^{2} f^{\prime 2}}{2}+\frac{r(2 k-f) f^{\prime}}{2}+2 f(k-f)\right) g^{\prime}\right. \\
& \left.-5\left(-\frac{3 r^{2}}{10}\left(-\frac{r f^{\prime}}{3}+k+\frac{2 f}{3}\right) f^{\prime \prime}-\frac{r^{2} f^{\prime 2}}{2}+\frac{r(k+7 f) f^{\prime}}{5}+(k-f)\left(k+\frac{6}{5} f\right)\right) g\right] .
\end{aligned}
$$

On the other hand, $N$ is constant again.

\subsection{Asymptotic solution}

As we did before for the asymptotically flat case, we have to determine first the asymptotic behavior of the solution. We assume that $f$ and $g$ can be expressed as a particular solution in the form of a $1 / r$ expansion, plus the general solution of the corresponding homogeneous equation:

$$
f(r)=f_{1 / r}(r)+f_{\mathrm{h}}(r), \quad g(r)=g_{1 / r}(r)+g_{\mathrm{h}}(r) .
$$

The large- $r$ expansions read

$$
\begin{aligned}
f_{1 / r} & =f_{\infty} \frac{r^{2}}{L^{2}}+k+\frac{2 M}{h^{\prime}\left(f_{\infty}\right) r}+\frac{42 M^{2} L^{2} \mu f_{\infty}}{\left[h^{\prime}\left(f_{\infty}\right)\right]^{3} r^{4}}+\frac{27 k M^{2} L^{4} \mu}{\left[h^{\prime}\left(f_{\infty}\right)\right]^{3} r^{6}}+\mathcal{O}\left(r^{-7}\right), \\
g_{1 / r}(r) & =-\frac{C}{h^{\prime}\left(f_{\infty}\right) r^{4}}-\frac{42 C M L^{2} \mu f_{\infty}}{\left[h^{\prime}\left(f_{\infty}\right)\right]^{3} r^{7}}+\frac{3 C M L^{4} \mu\left(23-3786 \mu f_{\infty}^{2}\right)}{\left[h^{\prime}\left(f_{\infty}\right)\right]^{5} r^{10}}+\mathcal{O}\left(r^{-12}\right) .
\end{aligned}
$$

On the other hand, the linearized homogeneous equations satisfied by $f_{\mathrm{h}}(r)$ and $g_{\mathrm{h}}(r)$ in the large- $r$ limit, read

$$
\frac{9 M f_{\infty} L^{2} \mu}{2 h^{\prime}\left(f_{\infty}\right)} f_{\mathrm{h}}^{\prime \prime}(r)+\frac{18 M L^{2} \mu f_{\infty}}{\left[h^{\prime}\left(f_{\infty}\right)\right] r} f_{\mathrm{h}}^{\prime}(r)-h^{\prime}\left(f_{\infty}\right) r f_{\mathrm{h}}(r)=0
$$

\footnotetext{
${ }^{7}$ This has been sometimes called characteristic polynomial, and it has been shown to be useful in characterizing the thermodynamic properties of black holes [13, 46, 65, 77, 78].
} 


$$
\frac{9 M f_{\infty} L^{2} \mu}{2 h^{\prime}\left(f_{\infty}\right)} g_{\mathrm{h}}^{\prime \prime}(r)+\frac{27 M f_{\infty} L^{2} \mu}{2 r h^{\prime}\left(f_{\infty}\right)} g_{\mathrm{h}}^{\prime}(r)-h^{\prime}\left(f_{\infty}\right) r g_{\mathrm{h}}(r)=0
$$

These equations are identical and have the following solutions

$$
\begin{aligned}
& f_{\mathrm{h}}(r) \sim A_{1} r^{-3 / 2} I_{1}\left(-\frac{2 \sqrt{2} r^{3 / 2} h^{\prime}\left(f_{\infty}\right)}{9 L \sqrt{M \mu f_{\infty}}}\right)+B_{1} r^{-3 / 2} K_{1}\left(\frac{2 \sqrt{2} r^{3 / 2} h^{\prime}\left(f_{\infty}\right)}{9 L \sqrt{M \mu f_{\infty}}}\right), \\
& g_{\mathrm{h}}(r) \sim \sim A_{2} r^{-3 / 2} I_{1}\left(-\frac{2 \sqrt{2} r^{3 / 2} h^{\prime}\left(f_{\infty}\right)}{9 L \sqrt{M \mu f_{\infty}}}\right)+B_{2} r^{-3 / 2} K_{1}\left(\frac{2 \sqrt{2} r^{3 / 2} h^{\prime}\left(f_{\infty}\right)}{9 L \sqrt{M \mu f_{\infty}}}\right)
\end{aligned}
$$

where $K_{\alpha}(x)$ and $I_{\alpha}(x)$ are modified Bessel functions. Thus, in order to guarantee a regular asymptotic limit we demand $\mu>0$, in which case by setting $A_{1}=A_{2}=0$ the homogeneous solutions decay faster than exponentially at infinity. ${ }^{8}$

In the limit of large- $r$, the behavior of $p(r)$ is accurately described by integrating just the particular solution $g_{1 / r}(r)$. This gives

$$
p(r)=-\frac{\Omega_{\infty}}{a}+\frac{C}{3\left[h^{\prime}\left(f_{\infty}\right)\right] r^{3}}+\frac{7 C \mu M L^{2} f_{\infty}}{\left[h^{\prime}\left(f_{\infty}\right)\right]^{3} r^{6}}+\frac{C \mu M^{2} L^{4}\left(-23+3786 \mu f_{\infty}^{2}\right)}{3\left[h^{\prime}\left(f_{\infty}\right)\right]^{5} r^{9}}+\mathcal{O}\left(r^{-11}\right)
$$

where $\Omega_{\infty}$ is a constant of integration which corresponds to the asymptotic angular velocity of the spacetime. From now on we will set $\Omega_{\infty}=0$. To interpret the integration constant $C$, we will require that our solution asymptotically approaches the slow rotation limit of the Kerr-AdS solution with appropriately rescaled cosmological length scale and ADM charges [79-83]:

$$
\begin{aligned}
d s^{2} \rightarrow & -\left(f_{\infty} \frac{r^{2}}{L^{2}}+k+\frac{2 M}{h^{\prime}\left(f_{\infty}\right) r}\right) d t^{2}+\frac{4 M a\left(1-k x^{2}\right)}{h^{\prime}\left(f_{\infty}\right) r} d t d \phi+\left(f_{\infty} \frac{r^{2}}{L^{2}}+k+\frac{2 M}{h^{\prime}\left(f_{\infty}\right) r}\right)^{-1} d r^{2} \\
& +\frac{r^{2} d x^{2}}{1-k x^{2}}+r^{2}\left(1-k x^{2}\right) d \phi^{2}
\end{aligned}
$$

Noting that $g_{t \phi} \sim-\left(1-k x^{2}\right) 2 J G_{\text {eff }} / r$, where $G_{\text {eff }}=-G / h^{\prime}\left(f_{\infty}\right)$, comparing this with our asymptotic expansion for $p(r)$ reveals

$$
C=6 M
$$

analogous to the asymptotically flat case, with $J=a M$.

\subsection{Near horizon solution}

The analysis of the near-horizon regime is completely analogous to the asymptotically flat case, so let us be brief. Near the horizon $r=r_{\mathrm{h}}$, the functions $f$ and $g$ are required to admit a series expansion of the form

$$
f(r)=4 \pi T\left(r-r_{\mathrm{h}}\right)+\sum_{n=2} a_{n}\left(r-r_{\mathrm{h}}\right)^{n}, \quad g(r)=\sum_{n=0} g_{n}\left(r-r_{\mathrm{h}}\right)^{n}
$$

\footnotetext{
${ }^{8}$ We always assume $h^{\prime}\left(f_{\infty}\right)<0$.
} 
for certain coefficients $a_{n}$ and $g_{n}$. Together with an infinity number of equations for these parameters, the equation for $f(92)$ yields two constraints between the mass, the temperature and the horizon radius: ${ }^{9}$

$$
\begin{aligned}
2 G M & =k r_{\mathrm{h}}+\frac{r_{\mathrm{h}}^{3}}{L^{2}}-\frac{3 k(4 \pi T)^{2} \mu L^{4}}{4 r_{\mathrm{h}}}-\frac{(4 \pi T)^{3} \mu L^{4}}{4}, \\
0 & =-k+4 \pi T r_{\mathrm{h}}-\frac{3 r_{\mathrm{h}}^{2}}{L^{2}}+\frac{3 k(4 \pi T)^{2} \mu L^{4}}{4 r_{\mathrm{h}}^{2}} .
\end{aligned}
$$

On the other hand, we find that all the coefficients $a_{n \geq 3}$ are determined by $a_{2}$. In the same way, the full sequence of coefficients $g_{n}$ contains only a free parameter which can be taken to be $g_{0}$. This is completely analogous to the asymptotically flat case. In particular, the few first relationships read

$$
\begin{aligned}
6 M & =g_{0}\left(\frac{3}{4} \mu L^{4}\left(2 k r_{\mathrm{h}}\left[(4 \pi T)-3 a_{2} r_{\mathrm{h}}\right]+(4 \pi T) r_{\mathrm{h}}^{2}\left[2 a_{2} r_{\mathrm{h}}-5(4 \pi T)\right]+10 k^{2}\right)+r_{\mathrm{h}}^{4}\right) \\
& -\frac{3}{4}(4 \pi T) g_{1} \mu L^{4} r_{\mathrm{h}}\left[(4 \pi T) r_{\mathrm{h}}+2 k\right] \\
0 & =g_{1}\left(\frac{3}{4} \mu L^{4}\left(-2 k r_{\mathrm{h}}\left[5 a_{2} r_{\mathrm{h}}+3(4 \pi T)\right]-(4 \pi T) r_{\mathrm{h}}^{2}\left[4 a_{2} r_{\mathrm{h}}+7(4 \pi T)\right]+10 k^{2}\right)+r_{\mathrm{h}}^{4}\right) \\
& +g_{0}\left(\frac{3}{2} \mu L^{4}\left(k\left[2(4 \pi T)-r_{\mathrm{h}}\left(9 a_{3} r_{\mathrm{h}}+4 a_{2}\right)\right]+r_{\mathrm{h}}\left[2 a_{2}^{2} r_{\mathrm{h}}^{2}+3(4 \pi T) r_{\mathrm{h}}\left(a_{3} r_{\mathrm{h}}-3 a_{2}\right)+2(4 \pi T)^{2}\right]\right)\right. \\
& \left.+4 r_{\mathrm{h}}^{3}\right)-3(4 \pi T) g_{2} \mu L^{4} r_{\mathrm{h}}^{2}\left[(4 \pi T) r_{\mathrm{h}}+2 k\right] .
\end{aligned}
$$

Thus, we need to fix the values of $a_{2}$ and $g_{0}$ in order to obtain a solution. These are fixed by requiring that the solution has the correct behavior at infinity that we determined above.

\subsection{Numerical solution}

The space of solutions in the AdS case is richer than in the flat case since the black holes depend now on two parameters: the mass and the cosmological constant. When the cosmological constant is zero, the corrections only depend on the dimensionless combination $\mu L^{4} / M^{2}$, and thus we only need to vary this parameter in order to sample the full space of solutions. In the AdS case, the corrections have two effects. On the one hand, they always become relevant for black holes of small masses, just like in the flat case - in fact, for small black holes the effect of the cosmological constant is irrelevant. On the other hand, the corrections change the structure of the vacuum, so the modifications to black holes can be important even if the black holes are not small. In fact, there is a maximum value for the parameter $\mu$ for which an AdS vacuum exists. The maximum value is $\mu_{\text {cr }}=\frac{4}{27}$, in whose case the AdS radius takes the value $\tilde{L}^{2}=\frac{2}{3} L^{2}$ and the theory is said to be at the critical point [37]. The critical limit is characterized by the divergence of the effective Newton's constant, which in general reads

$$
G_{\text {eff }}=\frac{G}{1-3 \mu f_{\infty}^{2}},
$$

\footnotetext{
${ }^{9}$ For a detailed analysis of the thermodynamic properties of static black holes, see [39].
} 

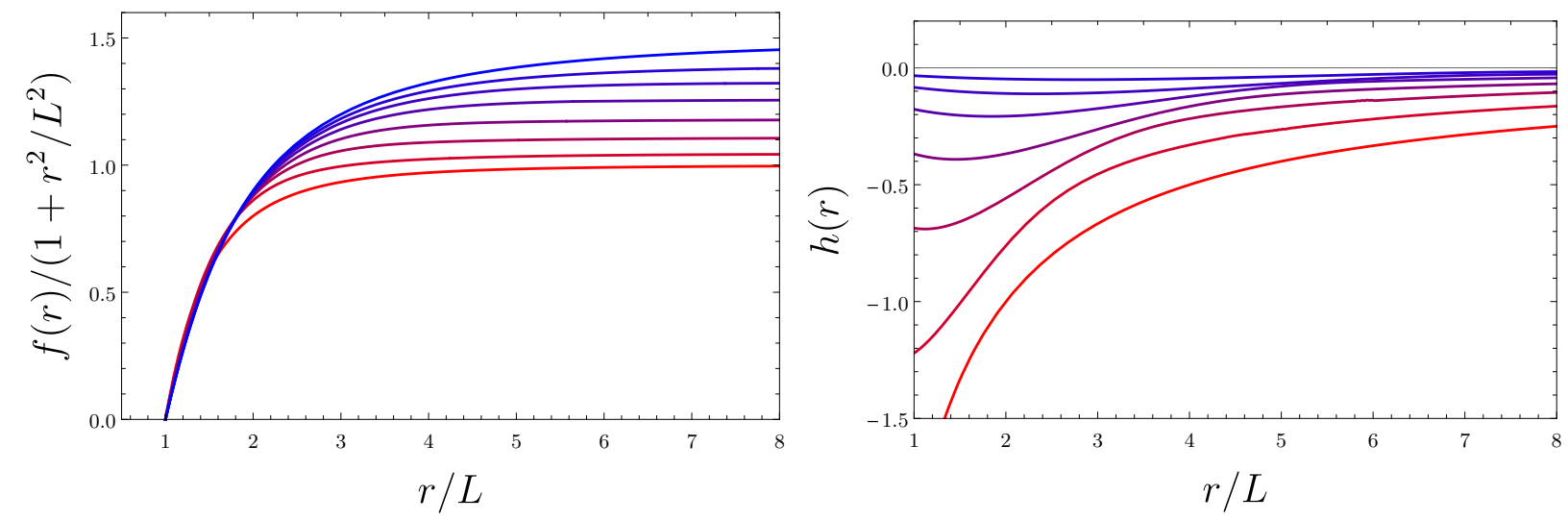

Figure 11: Rotating AdS black holes in Einsteinian cubic gravity. We show solutions with $r_{\mathrm{h}}=L$ and for $\mu=0,0.04,0.08,0.11,0.13,0.14,0.145,4 / 27$, with the $\mu=0$ Einstein solution the lowermost curves in red. Left: function $f(r)$ normalized by $1+r^{2} / L^{2}$. Right: function $h(r)=r^{2} p(r)$.

or equivalently by the vanishing of the linearized equations on AdS. Let us now show our results for the rotating black hole solutions.

In the small coupling limit, $\mu \ll 1$ and $\mu L^{4} / M^{4} \ll 1$, we can obtain an approximate solution by performing a perturbative expansion of the functions $f$ and $p$. The result reads

$$
\begin{aligned}
f(r) & =\frac{r^{2}}{L^{2}}+1-\frac{2 M}{r}+\mu\left[\frac{r^{2}}{L^{2}}-\frac{6 M}{r}-\frac{42 L^{2} M^{2}}{r^{4}}-\frac{27 L^{4} M^{2}}{r^{6}}+\frac{46 L^{4} M^{3}}{r^{7}}\right], \\
r^{2} p(r) & =-\frac{2 M}{r}+\mu\left[-\frac{6 M}{r}-\frac{42 L^{2} M^{2}}{r^{4}}+\frac{46 L^{4} M^{3}}{r^{7}}\right] .
\end{aligned}
$$

Let us note that the $\mathcal{O}\left(r^{2}\right)$ and $\mathcal{O}(1 / r)$ terms are related to the corrections to the AdS radius and to $G_{\text {eff. }}$. From these equations we can get for instance the mass and the angular velocity of the horizon as a function of the radius,

$$
\begin{aligned}
& M=\frac{r_{\mathrm{h}}\left(L^{2}+r_{\mathrm{h}}^{2}\right)}{2 L^{2}}+\mu\left[-\frac{27 r_{\mathrm{h}}^{3}}{8 L^{2}}-\frac{27 r_{\mathrm{h}}}{4}-\frac{27 L^{2}}{8 r_{\mathrm{h}}}-\frac{L^{4}}{2 r_{\mathrm{h}}^{3}}\right], \\
& \Omega=a\left[\frac{1}{L^{2}}+\frac{1}{r_{\mathrm{h}}^{2}}+\mu\left(\frac{1}{L^{2}}-\frac{27}{4 r_{\mathrm{h}}^{2}}-\frac{27 L^{2}}{2 r_{\mathrm{h}}^{4}}-\frac{27 L^{4}}{4 r_{\mathrm{h}}^{6}}\right)\right],
\end{aligned}
$$

perturbatively in $\mu$.

If we wish to study the solution for higher values of the coupling or for small masses, we need to resort to numerical methods. Equations (92) and (93) can be solved numerically by imposing the boundary conditions described in the previous subsection. For illustration purposes, let us study how the corrections modify the black hole solutions when we fix the size of the horizon while varying $\mu$ from zero to the critical value. In Fig. 11 we show the numerical solution for black holes of radius $r_{\mathrm{h}}=L$. The main effect of the corrections on the function $f$ is to change its asymptotic behavior due to the different value of the AdS radius. On the other hand, in the case of the function $h$, we see that its value gets smaller as we increase $\mu$. Let us recall that in the metric this function appears multiplied by $a=J / M$. Therefore, when we increase $\mu$ leaving $a$ and $r_{\mathrm{h}}$ fixed, the effect of the angular momentum becomes less and less relevant. In the critical limit $\mu \rightarrow 4 / 27$ the solution 


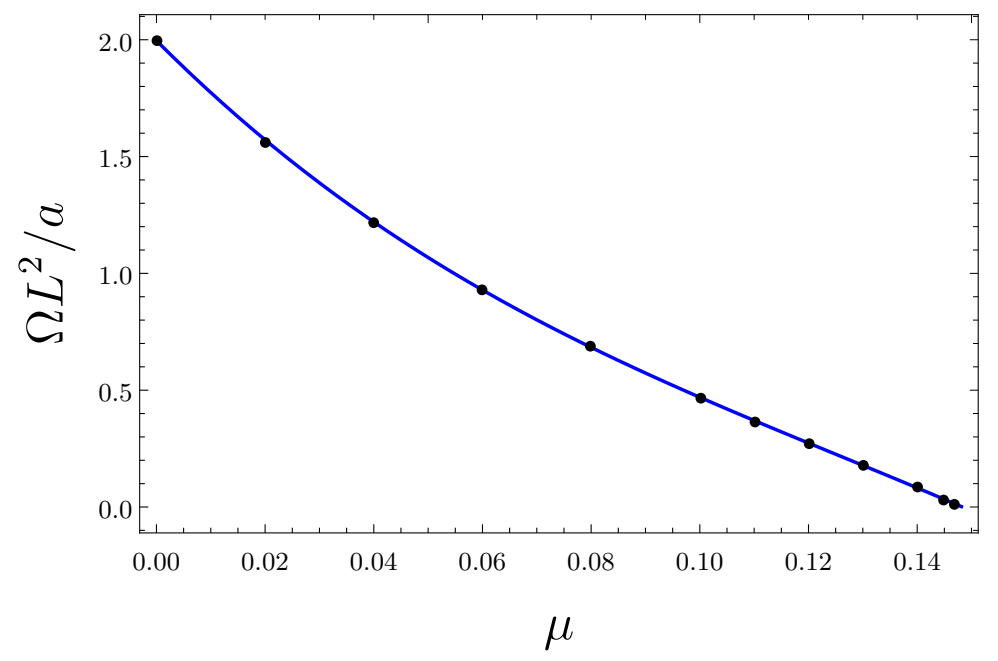

Figure 12: Angular velocity of the horizon as a function of $\mu$ for black holes of radius $r_{\mathrm{h}}=L$. The points represent numerical data while blue curve is a fitting polynomial of degree 4 .

seems to tend to $h \rightarrow 0$, so that no (regular) rotating solution exists in that case. Let us note that when we approach the critical limit leaving the radius fixed, the mass goes to zero, so it means that we can have slowly rotating black holes with $a \gg M$.

The irrelevance of rotation as we increase $\mu$ is better illustrated by looking at the angular velocity, which we show in Fig. 12. As we can see, $\Omega L^{2} / a$ goes to zero as we approach the critical limit. This is a quite exotic behavior, since in the case of Einstein gravity this quantity is bounded from below according to $\Omega L^{2} / a>1$. Now, regarding the absolute value of the angular velocity $\Omega$ and not the ratio $\Omega / a$, if we considered black holes of fixed $J$ and took the critical limit, we would find that $\Omega$ diverges. However, it seems to make more sense to fix $a$, so that the total angular momentum $J=a M$ goes to zero in the same way as the mass. In that case, the angular velocity tends to zero in the critical limit and there are, in fact, no rotating solutions.

\subsection{Spinning solutions in the critical limit}

As we have just seen, the critical theory is special, as it seems to allow only for massless and spinless black hole solutions. However, one can have solutions with non-vanishing charges if the condition of regularity is dropped. In particular, small-mass black holes in the critical theory were studied in Ref. [37], where they were found to develop a singularity at the horizon. Here, we are going to study rotating massless black holes.

At the critical point, $\mu=4 / 27$, the equation (92) with $M=0$ is solved by [37]

$$
f(r)=\frac{3\left(r^{2}-r_{h}^{2}\right)}{2 L^{2}}
$$

Thus, when $a=0$, the metric (91) represents that of a static black hole with a regular horizon placed at $r=r_{\mathrm{h}}$. Let us then consider the effect of rotation. After some manipulations, equation 


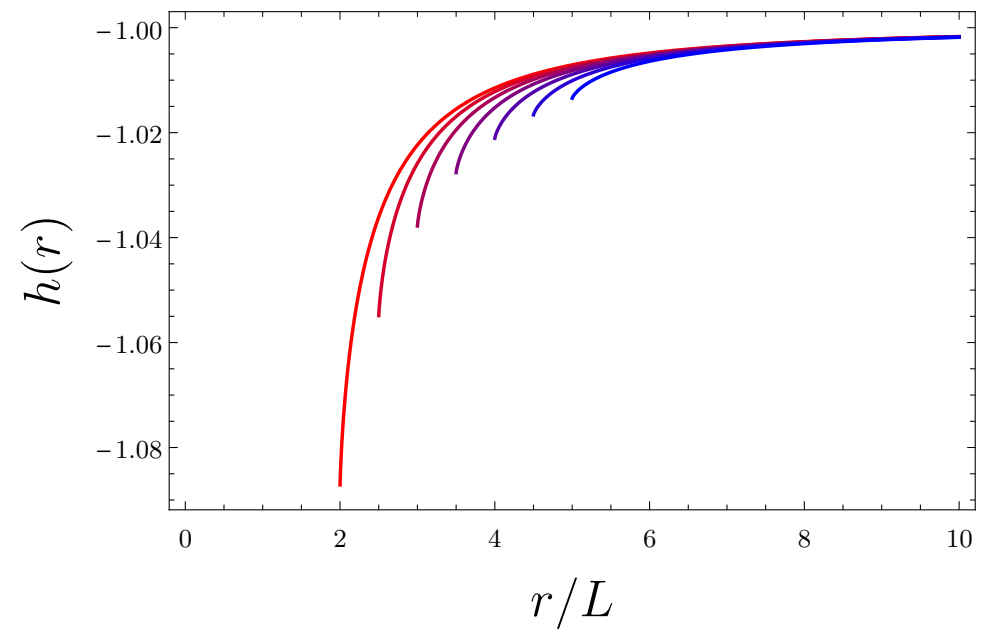

Figure 13: Function $h(r)=r^{2} p(r)$ in the critical limit for several values of $r_{\mathrm{h}}$, corresponding to the endpoint of each curve. From red to blue we have $r_{\mathrm{h}} / L=2,2.5,3,3.5,4,4.5,5$.

(93) can be written as

$$
\tilde{C}+\left(\frac{10 L^{2}}{3}-6 r_{\mathrm{h}}^{2}\right) g-\frac{d}{d r}\left[r^{2}\left(r^{2}-r_{\mathrm{h}}^{2}\right) \frac{d g}{d r}\right]=0
$$

where $\tilde{C}$ is an integration constant related to $C$. The general solution to this equation can be expressed in several ways in terms of hypergeometric functions, but the most useful way is the following one:

$$
g(r)=-\frac{\tilde{C}}{\left(\frac{10 L^{2}}{3}-6 r_{\mathrm{h}}^{2}\right)}+c_{12} F_{1}\left(1-\alpha, \alpha-\frac{3}{2} ;-\frac{1}{2} ; \frac{r_{\mathrm{h}}^{2}}{r^{2}}\right)+\frac{c_{2}}{r^{3}}{ }_{2} F_{1}\left(\frac{5}{2}-\alpha, \alpha ; \frac{5}{2} ; \frac{r_{\mathrm{h}}^{2}}{r^{2}}\right)
$$

where

$$
\alpha=\frac{5}{4}\left(1+\sqrt{1-\frac{8 L^{2}}{15 r_{\mathrm{h}}^{2}}}\right) .
$$

Now, the integration constants are fixed by analyzing the asymptotic behavior at infinity, which reads

$$
g(r)=-\frac{\tilde{C}}{\left(\frac{10 L^{2}}{3}-6 r_{\mathrm{h}}^{2}\right)}+c_{1}-\left(\frac{10 L^{2}}{3}-6 r_{\mathrm{h}}^{2}\right) \frac{c_{1}}{2 r^{2}}+\frac{c_{2}}{r^{3}}+\ldots
$$

Now, in terms of the function $h(r)=r^{2} p(r)$, the asymptotic expansion becomes

$$
h(r)=r^{3}\left(-\frac{\tilde{C}}{\left(\frac{10 L^{2}}{3}-6 r_{\mathrm{h}}^{2}\right)}+c_{1}\right)+\left(\frac{10 L^{2}}{3}-6 r_{\mathrm{h}}^{2}\right) \frac{c_{1} r}{2}-\frac{c_{2}}{2}+\ldots .
$$

Since the $r^{3}$ and $r$ divergent terms are not desired, we must set $c_{1}=\tilde{C}=0$. In that case, the behavior of $h$ at infinity is $h=-c_{2} / 2+\mathcal{O}(1 / r)$, so that it necessarily tends to a non-vanishing value. Although this might seem strange, this is similar to what happens to the function $f(r)$ in the critical limit, which instead of behaving as $f(r)=f_{\mathrm{AdS}}(r)+\mathcal{O}(1 / r)$ it goes as $f(r)=f_{\mathrm{AdS}}(r)+\mathcal{O}(1)$. Thus, 


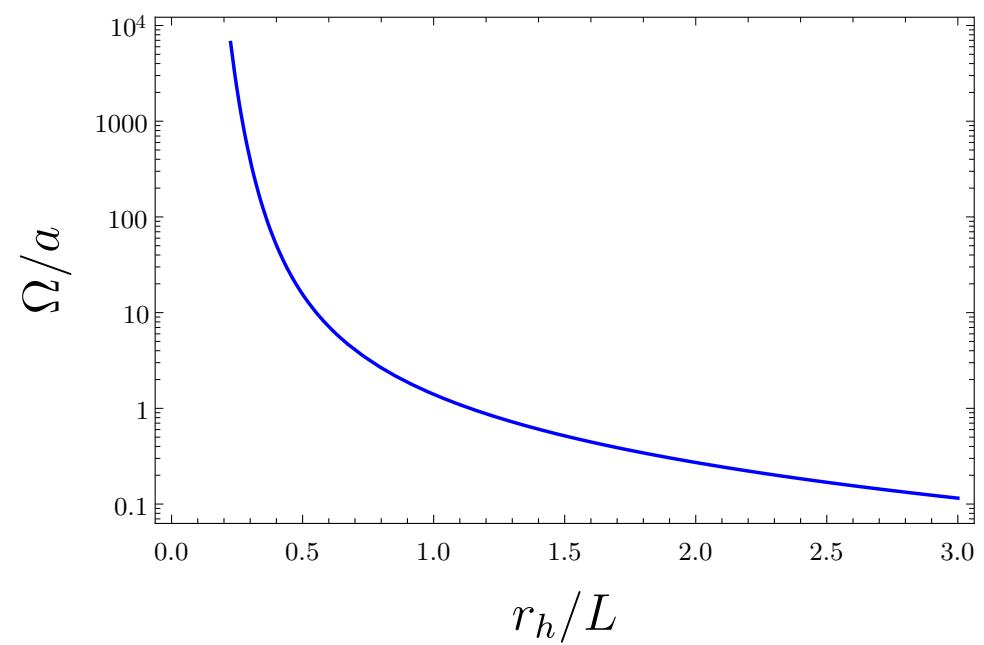

Figure 14: Angular velocity for the critical rotating black holes as a function of the radius of the horizon.

let us also set $c_{2}=2$, so that the metric component $g_{t \phi}$ takes the asymptotic value $g_{t \phi} \rightarrow-a \sin ^{2} \theta$. In this case, $a$ is a parameter that controls the angular momentum, although it no longer can be interpreted as the angular momentum per mass. The final expression for $h(r)$ after integration of $g(r)$ reads

$$
h(r)=-\frac{r^{2}}{\left(r_{\mathrm{h}}^{2}-\frac{5 L^{2}}{9}\right)}\left[1-{ }_{2} F_{1}\left(\frac{3}{2}-\alpha, \alpha-1 ; \frac{3}{2} ; \frac{r_{\mathrm{h}}^{2}}{r^{2}}\right)\right] .
$$

It can be checked that this expression is real and regular for any value of $r_{\mathrm{h}}$ - in particular, the limit $r_{\mathrm{h}} \rightarrow \sqrt{5} / 3 L$ is finite. In Fig. 13 we show the profile of $h(r)$ for several values of $r_{\mathrm{h}}$, where we can see that $h(r)$ is finite everywhere. However, $h$ is not smooth at $r=r_{\mathrm{h}}$, since its expansion near the horizon contains terms such as $\left(r-r_{\mathrm{h}}\right) \log \left(1-r / r_{\mathrm{h}}\right)$, so that its derivatives are divergent.

The value of $h\left(r_{\mathrm{h}}\right)$ is rapidly growing as we decrease $r_{\mathrm{h}}$, and as a consequence, the angular velocity $\Omega=-a h\left(r_{\mathrm{h}}\right) / r_{\mathrm{h}}^{2}$, also becomes very high. The angular velocity is given by

$$
\Omega=\frac{a}{\left(r_{\mathrm{h}}^{2}-\frac{5 L^{2}}{9}\right)}\left(1-\frac{\sqrt{\pi}}{2 \Gamma\left(\frac{5}{2}-\alpha\right) \Gamma(\alpha)}\right),
$$

and we plot it in Fig. 14, where we observe that it diverges exponentially for $r_{\mathrm{h}} \rightarrow 0$. Obviously, the slowly rotating approximation is not valid when $\Omega$ is large, so we have to restrict to sufficiently large values of $r_{\mathrm{h}}$.

\section{$5 \quad$ Final comments}

In this paper we have constructed the slowly rotating black hole solutions of Einsteinian cubic gravity both with flat and AdS asymptotes and studied some of their properties. A summary of our results can be found in the introduction. Let us close with some final comments, mostly regarding possible future explorations. 
In the static case, the order-reduction phenomenon observed in the equations of motion of ECG turns out to be a general property of GQT gravities in general dimensions and orders. Here we have observed a similar mechanism taking place in the slowly rotating case. It would be interesting to explore whether this is a common feature of this general class of theories.

While the slowly rotating black holes of Lovelock theories have been already characterized see appendix A - no studies of the kind have been performed, to our knowledge, in the case of Quasi-topological gravities. It would be of course interesting to fill this gap. Moreover, as described in the appendix, the slowly rotating solutions in the Lovelock case are completely characterized by the metric for the static solution. This is reminiscent of the Newman-Janis algorithm and it could be interesting to better understand this feature. On the other hand, the slowly rotating solutions in ECG are not so obviously connected with the properties of the static solution.

In the present paper we have not given much attention to the thermodynamic properties of the solutions, the reason being that the effect of the angular momentum on the entropy and the temperature only appears at order $\mathcal{O}\left(a^{2}\right)$, and hence it is not captured by the leading-order solution. Nevertheless, it would be interesting to perform this kind of study, particularly from a holographic perspective.

Finally, a study of the solutions including arbitrary values of the rotation parameter would be perhaps worth pursuing, but a priori considerably more challenging.

\section{Acknowledgements}

We thank José Edelstein and Julio Oliva for useful discussions. The work of PB was supported by the Simons foundation through the It From Qubit Simons collaboration. PAC was supported by the KU Leuven grant "Bijzonder Onderzoeksfonds C16/16/005 - Horizons in hoge-energie fysica". The work of RBM and RAH is supported by the Natural Sciences and Engineering Research Council of Canada, the latter through the Banting Postdoctoral Fellowship program. At least some of us were also supported physically by planet Earth through the electromagnetic and gravitational interactions.

\section{A Slowly rotating black holes in Lovelock gravities}

In this appendix we review the single-axis slowly rotating black holes of Lovelock theories in general dimensions.

\section{A.1 Einstein gravity}

Let us start considering $D$-dimensional Einstein gravity with a negative cosmological constant,

$$
I=\frac{1}{16 \pi G} \int d^{D} x \sqrt{|g|}\left[\frac{(D-1)(D-2)}{L^{2}}+R\right] .
$$


Assuming a single axis of rotation, the slowly rotating version of the Kerr-AdS solution - also known as Myers-Perry black hole in its more general form for $D \geq 5$ - can be written as

$$
\begin{aligned}
d s^{2}= & -\left[k+\frac{r^{2}}{L^{2}} g(r)\right] d t^{2}+\frac{d r^{2}}{\left[k+\frac{r^{2}}{L^{2}} g(r)\right]}+\frac{2 a r^{2}}{L^{2}} g(r)\left(1-k x^{2}\right) d t d \phi \\
& +r^{2}\left[\frac{d x^{2}}{\left(1-k x^{2}\right)}+\left(1-k x^{2}\right) d \phi^{2}+x^{2} d \sigma_{(D-4)}^{2}\right]
\end{aligned}
$$

where:

$$
x \equiv \cos \theta \quad \text { for } \quad k=1 ; \quad x \equiv \cosh \theta \quad \text { for } \quad k=-1 .
$$

In the second line of eq. (122), $r^{2}$ multiplies the metric of $\mathbb{S}^{(D-2)}, \mathbb{R}^{(D-2)}$ and $\mathbb{H}^{(D-2)}$ for $k=0,1,-1$ respectively, which in the coordinates used above means that $d \sigma_{(D-4)}^{2}$ is the metric of a round $\mathbb{S}^{(D-4)}$ for $k= \pm 1$, and $d \sigma_{(D-4)}^{2} \equiv d \vec{y}_{(D-4)}^{2} / L^{2}$, with $d \vec{y}_{(D-4)}^{2}$ the metric of $(D-4)$-dimensional Euclidean space for $k=0$.

Observe that the same function $g(r)$ which characterizes the static limit of the solution appears in the only new components arising at order $\mathcal{O}(a)$ - namely, $g_{t \phi}=g_{\phi t}$. The function $g(r)$ is given by

$$
g(r)=\left[1-\frac{16 \pi G M L^{2}}{(D-2) \Omega_{(D-2)} r^{(D-1)}}\right] \Rightarrow-g_{t t}=g_{r r}^{-1}=\left[k-\frac{16 \pi G M}{(D-2) \Omega_{(D-2)} r^{(D-3)}}+\frac{r^{2}}{L^{2}}\right] .
$$

In the above expression, $M$ is the ADM mass of the solution, and $\Omega_{(D-2)} \equiv 2 \pi^{\frac{(D-1)}{2}} / \Gamma\left[\frac{D-1}{2}\right]$ is the area of $\mathbb{S}^{(D-2)}$. Naturally, when $a \rightarrow 0$, eq. (122) simply reduces to the usual Schwarzschild-AdS solution with various horizon geometries.

Another convenient set of coordinates is found by setting $\phi \rightarrow \phi-a t / L^{2}$, which replaces

$$
2 g_{t \phi} \rightarrow 2 g_{t \phi}-\frac{2 a r^{2}}{L^{2}}\left(1-k x^{2}\right)=\frac{2 a r^{2}}{L^{2}}[g(r)-1]\left(1-k x^{2}\right)=\frac{-32 \pi G M\left(1-k x^{2}\right)}{(D-2) \Omega_{(D-2)} r^{(D-3)}} .
$$

The advantage of these coordinates is that the asymptotic angular speed of the solution vanishes,

$$
\Omega_{\infty} \equiv \lim _{r \rightarrow \infty} \frac{-g_{t \phi}}{g_{\phi \phi}}=\lim _{r \rightarrow \infty} \frac{16 \pi G M}{(D-2) \Omega_{(D-2)} r^{(D-1)}}=0
$$

for all $D$.

A way to understand the appearance of the Schwarzschild-AdS blackening factor $g(r)$ in the $g_{t \phi}=g_{\phi t}$ components which will turn out to be useful when we turn on the higher-order Lovelock couplings is the following. First, note that the metric eq. (122) in the $k=0$ case is actually related to the $a=0$ metric by a coordinate transformation. In particular, applying $t \rightarrow t-a \phi$ to the Schwarzschild-AdS black brane produces eq. (122) at order $\mathcal{O}(a)$. While this is no longer the case in the $k= \pm 1$ cases, if we consider an ansatz of the form eq. (122) with $g(r)$ replaced by some other function $p(r)$ in the $g_{t \phi}=g_{\phi t}$ components, once $g(r)$ is determined using the $\mathcal{O}\left(a^{0}\right)$ equations, the only components of Einstein's equations which are modified at order $\mathcal{O}\left(a^{1}\right)$ - namely, $\mathcal{E}_{t \phi}=\mathcal{E}_{\phi t}=0$ - do not depend on $k$ explicitly. Therefore, the fact that $p(r)=g(r)$ holds for $k=0$ - which is just a consequence of the existence of the coordinate transformation relating the rotating and static black brane solutions - implies that it also holds for the less trivial cases $k= \pm 1$. A similar phenomenon occurs for Lovelock gravities, as we explain now. 


\section{A.2 Lovelock gravities}

Let us now consider a generic $D$-dimensional Lovelock gravity $[1,2]$, whose action can be written as

$$
I=\frac{1}{16 \pi G} \int d^{D} x \sqrt{|g|}\left[\frac{(D-1)(D-2)}{L^{2}}+R+\sum_{n=2}^{\lfloor D / 2\rfloor} \frac{\lambda_{2 n} L^{2(n-1)}(D-2) !}{(D-2 n) !} \mathcal{X}_{2 n}\right],
$$

where $\lambda_{2 n}$ are dimensionless couplings and $\mathcal{X}_{2 n}$ are the dimensionally-extended Euler densities of $2 n$-dimensional manifolds - e.g., $\mathcal{X}_{4} \equiv R^{2}-4 R_{a b} R^{a b}+R_{a b c d} R^{a b c d}$ is the usual Gauss-Bonnet density.

The static black hole solutions of eq. (127) have been extensively studied in the literature see e.g., [3-6, 9, 11-14]. Generalizations of the slowly rotating Kerr-AdS solution appearing in eqs. (122) and (124) exist for this class of theories. Just like in the Einstein gravity case, these solutions are characterized by the static-solution blackening-factor, namely, they also take the form eq. (122), where now $g(r)$ is determined by the algebraic equation

$$
1-g(r)+\sum_{n=2}^{\lfloor D / 2\rfloor} \lambda_{2 n} g(r)^{n}=\frac{16 \pi G M L^{2}}{(D-2) \Omega_{(D-2)} r^{(D-1)}} .
$$

For example, including the Gauss-Bonnet density alone, one finds

$$
g(r)=\frac{1}{2 \lambda_{4}}\left[1 \mp \sqrt{1-4 \lambda_{4}+\frac{64 \pi G M \lambda_{4} L^{2}}{(D-2) \Omega_{(D-2)} r^{(D-1)}}}\right] .
$$

The slowly rotating solutions including the Gauss-Bonnet and cubic Lovelock densities were constructed for generic $k$ in [63] and [64] respectively. For $k=0$, the full boosted black branes (with arbitrarily large values of $a$ ) were obtained in $[84,85]$. The general slowly rotating case was studied in [65].

The mechanism explained at the end of the previous subsection regarding the $k$-independence of the equations of motion and the role played by the function $g(r)$ in the slowly rotating solutions holds for the general Lovelock theory in eq. (127), which therefore possesses solutions of the form eq. (122) with $g(r)$ determined by eq. (128).

\section{References}

[1] D. Lovelock, Divergence-free tensorial concomitants, aequationes mathematicae 4 (1970) $127-138$.

[2] D. Lovelock, The Einstein tensor and its generalizations, J. Math. Phys. 12 (1971) 498-501.

[3] J. T. Wheeler, Symmetric Solutions to the Gauss-Bonnet Extended Einstein Equations, Nucl. Phys. B268 (1986) 737-746.

[4] J. T. Wheeler, Symmetric Solutions to the Maximally Gauss-Bonnet Extended Einstein Equations, Nucl. Phys. B273 (1986) 732. 
[5] D. G. Boulware and S. Deser, String Generated Gravity Models, Phys. Rev. Lett. 55 (1985) 2656 .

[6] R. C. Myers and J. Z. Simon, Black Hole Thermodynamics in Lovelock Gravity, Phys. Rev. D38 (1988) 2434-2444.

[7] R.-G. Cai and K.-S. Soh, Topological black holes in the dimensionally continued gravity, Phys. Rev. D59 (1999) 044013, [gr-qc/9808067].

[8] R. Aros, R. Troncoso and J. Zanelli, Black holes with topologically nontrivial AdS asymptotics, Phys. Rev. D63 (2001) 084015, [hep-th/0011097].

[9] R.-G. Cai, Gauss-Bonnet black holes in AdS spaces, Phys. Rev. D65 (2002) 084014, [hep-th/0109133].

[10] M. Dehghani and R. B. Mann, NUT-charged black holes in Gauss-Bonnet gravity, Phys. Rev. D 72 (2005) 124006, [hep-th/0510083].

[11] M. H. Dehghani and R. Pourhasan, Thermodynamic instability of black holes of third order Lovelock gravity, Phys. Rev. D79 (2009) 064015, [0903.4260].

[12] J. de Boer, M. Kulaxizi and A. Parnachev, Holographic Lovelock Gravities and Black Holes, JHEP 06 (2010) 008, [0912.1877].

[13] X. O. Camanho and J. D. Edelstein, A Lovelock black hole bestiary, Class. Quant. Grav. 30 (2013) 035009, [1103.3669].

[14] C. Garraffo and G. Giribet, The Lovelock Black Holes, Mod. Phys. Lett. A23 (2008) 1801-1818, [0805.3575].

[15] J. Oliva and S. Ray, A new cubic theory of gravity in five dimensions: Black hole, Birkhoff's theorem and C-function, Class. Quant. Grav. 27 (2010) 225002, [1003.4773].

[16] R. C. Myers and B. Robinson, Black Holes in Quasi-topological Gravity, JHEP 08 (2010) 067, [1003.5357].

[17] J. Oliva and S. Ray, Birkhoff's Theorem in Higher Derivative Theories of Gravity, Class. Quant. Grav. 28 (2011) 175007, [1104.1205].

[18] J. Oliva and S. Ray, Birkhoff's Theorem in Higher Derivative Theories of Gravity II, Phys. Rev. D86 (2012) 084014, [1201.5601].

[19] M. H. Dehghani, A. Bazrafshan, R. B. Mann, M. R. Mehdizadeh, M. Ghanaatian and M. H. Vahidinia, Black Holes in Quartic Quasitopological Gravity, Phys. Rev. D85 (2012) 104009, [1109.4708].

[20] A. Cisterna, L. Guajardo, M. Hassaine and J. Oliva, Quintic quasi-topological gravity, JHEP 04 (2017) 066, [1702.04676].

[21] S. Ray, Birkhoff's theorem in Lovelock gravity for general base manifolds, Class. Quant. Grav. 32 (2015) 195022, [1505.03830].

[22] R. A. Hennigar, D. Kubiznak and R. B. Mann, Generalized quasitopological gravity, Phys. Rev. D95 (2017) 104042, [1703.01631]. 
[23] P. Bueno and P. A. Cano, On black holes in higher-derivative gravities, Class. Quant. Grav. 34 (2017) 175008, [1703.04625].

[24] J. Ahmed, R. A. Hennigar, R. B. Mann and M. Mir, Quintessential Quartic Quasi-topological Quartet, JHEP 05 (2017) 134, [1703.11007].

[25] P. Bueno and P. A. Cano, Universal black hole stability in four dimensions, Phys. Rev. D96 (2017) 024034, [1704.02967].

[26] P. Bueno, P. A. Cano and R. A. Hennigar, (Generalized) quasi-topological gravities at all orders, Class. Quant. Grav. 37 (2020) 015002, [1909.07983].

[27] P. Bueno, P. A. Cano, J. Moreno and A. Murcia, All higher-curvature gravities as Generalized quasi-topological gravities, JHEP 11 (2019) 062, [1906.00987].

[28] R. A. Hennigar, Criticality for charged black branes, JHEP 09 (2017) 082, [1705.07094].

[29] M. Mir and R. B. Mann, On generalized quasi-topological cubic-quartic gravity: thermodynamics and holography, JHEP 07 (2019) 012, [1902.10906].

[30] M. Mir, R. A. Hennigar, J. Ahmed and R. B. Mann, Black hole chemistry and holography in generalized quasi-topological gravity, JHEP 08 (2019) 068, [1902.02005].

[31] Y.-Z. Li, H.-S. Liu and H. Lu, Quasi-Topological Ricci Polynomial Gravities, JHEP 02 (2018) 166, [1708.07198].

[32] Y.-Z. Li, H. Lu and J.-B. Wu, Causality and a-theorem Constraints on Ricci Polynomial and Riemann Cubic Gravities, Phys. Rev. D97 (2018) 024023, [1711.03650].

[33] Y.-Z. Li, H. Lu and Z.-F. Mai, Universal Structure of Covariant Holographic Two-Point Functions In Massless Higher-Order Gravities, JHEP 10 (2018) 063, [1808.00494].

[34] P. Bueno and P. A. Cano, Einsteinian cubic gravity, Phys. Rev. D94 (2016) 104005, [1607.06463].

[35] P. Bueno and P. A. Cano, Four-dimensional black holes in Einsteinian cubic gravity, Phys. Rev. D94 (2016) 124051, [1610.08019].

[36] R. A. Hennigar and R. B. Mann, Black holes in Einsteinian cubic gravity, Phys. Rev. D95 (2017) 064055, [1610.06675].

[37] X.-H. Feng, H. Huang, Z.-F. Mai and H. Lu, Bounce Universe and Black Holes from Critical Einsteinian Cubic Gravity, Phys. Rev. D96 (2017) 104034, [1707.06308].

[38] R. A. Hennigar, M. B. J. Poshteh and R. B. Mann, Shadows, Signals, and Stability in Einsteinian Cubic Gravity, Phys. Rev. D97 (2018) 064041, [1801.03223].

[39] P. Bueno, P. A. Cano and A. Ruipérez, Holographic studies of Einsteinian cubic gravity, JHEP 03 (2018) 150, [1802.00018].

[40] M. B. J. Poshteh and R. B. Mann, Gravitational Lensing by Black Holes in Einsteinian Cubic Gravity, Phys. Rev. D 99 (2019) 024035, [1810.10657].

[41] W. T. Emond and N. Moynihan, Scattering Amplitudes, Black Holes and Leading Singularities in Cubic Theories of Gravity, JHEP 12 (2019) 019, [1905.08213]. 
[42] J. Jiang and B. Deng, Investigating the holographic complexity in Einsteinian cubic gravity, Eur. Phys. J. C 79 (2019) 832.

[43] M. R. Mehdizadeh and A. H. Ziaie, Traversable wormholes in Einsteinian cubic gravity, Mod. Phys. Lett. A 35 (2019) 2050017, [1903.10907].

[44] A. M. Frassino and J. V. Rocha, Charged black holes in Einsteinian cubic gravity and non-uniqueness, 2002.04071.

[45] M. Kord Zangeneh and A. Kazemi, Topological Born-Infeld charged black holes in Einsteinian cubic gravity, 2003.04458.

[46] P. Bueno, P. A. Cano, R. A. Hennigar, V. A. Penas and A. Ruiperez, Partition functions on slightly squashed spheres and flux parameters, 2001.10020.

[47] G. Arciniega, J. D. Edelstein and L. G. Jaime, Towards geometric inflation: the cubic case, Phys. Lett. B802 (2020) 135272, [1810.08166].

[48] A. Cisterna, N. Grandi and J. Oliva, On four-dimensional Einsteinian gravity, quasitopological gravity, cosmology and black holes, 1811.06523.

[49] G. Arciniega, P. Bueno, P. A. Cano, J. D. Edelstein, R. A. Hennigar and L. G. Jaime, Geometric Inflation, Phys. Lett. B802 (2020) 135242, [1812.11187].

[50] C. Erices, E. Papantonopoulos and E. N. Saridakis, Cosmology in cubic and $f(P)$ gravity, Phys. Rev. D 99 (2019) 123527, [1903.11128].

[51] M. Marciu, A note on the dynamical features for the extended $f(P)$ cubic gravity, 2003.06403.

[52] I. Quiros, R. Garcia-Salcedo, T. Gonzalez, J. L. M. Martinez and U. Nucamendi, Global asymptotic dynamics of Cosmological Einsteinian Cubic Gravity, 2003.10516.

[53] G. Arciniega, L. Jaime and G. Piccinelli, Inflationary predictions of Geometric Inflation, 2001.11094.

[54] M. C. Pookkillath, A. De Felice and A. A. Starobinsky, Anisotropic instability in a higher order gravity theory, 2004.03912.

[55] M. Marciu, Dynamical aspects for scalar fields coupled to cubic contractions of the Riemann tensor, 2004.07120.

[56] H. Lu, A. Perkins, C. N. Pope and K. S. Stelle, Spherically Symmetric Solutions in Higher-Derivative Gravity, Phys. Rev. D92 (2015) 124019, [1508.00010].

[57] H. Lu, A. Perkins, C. N. Pope and K. S. Stelle, Black Holes in Higher-Derivative Gravity, Phys. Rev. Lett. 114 (2015) 171601, [1502.01028].

[58] S. Deser, O. Sarioglu and B. Tekin, Spherically symmetric solutions of Einstein + non-polynomial gravities, Gen. Rel. Grav. 40 (2008) 1-7, [0705.1669].

[59] A. Colleaux, Regular black hole and cosmological spacetimes in Non-Polynomial Gravity theories. PhD thesis, Trento U., 2019-06-17. 
[60] R. C. Myers and M. J. Perry, Black Holes in Higher Dimensional Space-Times, Annals Phys. 172 (1986) 304.

[61] A. Anabalon, N. Deruelle, Y. Morisawa, J. Oliva, M. Sasaki, D. Tempo et al., Kerr-Schild ansatz in Einstein-Gauss-Bonnet gravity: An exact vacuum solution in five dimensions, Class. Quant. Grav. 26 (2009) 065002, [0812.3194].

[62] M. Cvetic, X.-H. Feng, H. Lu and C. N. Pope, Rotating Solutions in Critical Lovelock Gravities, Phys. Lett. B765 (2017) 181-187, [1609.09136].

[63] H.-C. Kim and R.-G. Cai, Slowly Rotating Charged Gauss-Bonnet Black holes in AdS Spaces, Phys. Rev. D77 (2008) 024045, [0711.0885].

[64] R. Yue, D. Zou, T. Yu, P. Li and Z. Yang, Slowly rotating charged black holes in anti-de Sitter third order Lovelock gravity, Gen. Rel. Grav. 43 (2011) 2103-2114, [1011.5293].

[65] X. O. Camanho, Lovelock gravity, black holes and holography. PhD thesis, Santiago de Compostela U., 2013. 1509.08129.

[66] Y. Brihaye and E. Radu, Five-dimensional rotating black holes in Einstein-Gauss-Bonnet theory, Phys. Lett. B661 (2008) 167-174, [0801.1021].

[67] Y. Brihaye, B. Kleihaus, J. Kunz and E. Radu, Rotating black holes with equal-magnitude angular momenta in d=5 Einstein-Gauss-Bonnet theory, JHEP 11 (2010) 098, [1010.0860].

[68] V. Cardoso, M. Kimura, A. Maselli and L. Senatore, Black Holes in an Effective Field Theory Extension of General Relativity, Phys. Rev. Lett. 121 (2018) 251105, [1808.08962].

[69] P. A. Cano and A. Ruiperez, Leading higher-derivative corrections to Kerr geometry, JHEP 05 (2019) 189, [1901.01315].

[70] H. S. Reall and J. E. Santos, Higher derivative corrections to Kerr black hole thermodynamics, JHEP 04 (2019) 021, [1901.11535].

[71] P. A. Cano and D. Pereniguez, Extremal Rotating Black Holes in Einsteinian Cubic Gravity, Phys. Rev. D101 (2020) 044016, [1910.10721].

[72] D. J. Burger, W. T. Emond and N. Moynihan, Rotating Black Holes in Cubic Gravity, 1910.11618.

[73] P. Bueno, P. A. Cano, R. A. Hennigar and R. B. Mann, NUTs and bolts beyond Lovelock, JHEP 10 (2018) 095, [1808.01671].

[74] LIGO Scientific, Virgo collaboration, B. Abbott et al., Observation of Gravitational Waves from a Binary Black Hole Merger, Phys. Rev. Lett. 116 (2016) 061102, [1602.03837].

[75] B. Mashhoon, Stability of charged rotating black holes in the eikonal approximation, Phys. Rev. D31 (1985) 290-293.

[76] V. Cardoso, A. S. Miranda, E. Berti, H. Witek and V. T. Zanchin, Geodesic stability, Lyapunov exponents and quasinormal modes, Phys. Rev. D79 (2009) 064016, [0812.1806].

[77] X. O. Camanho, J. D. Edelstein and M. F. Paulos, Lovelock theories, holography and the fate of the viscosity bound, JHEP 05 (2011) 127, [1010.1682]. 
[78] M. F. Paulos, Holographic phase space: c-functions and black holes as renormalization group flows, JHEP 05 (2011) 043, [1101.5993].

[79] S. Deser and B. Tekin, Energy in generic higher curvature gravity theories, Phys. Rev. D67 (2003) 084009, [hep-th/0212292].

[80] R. L. Arnowitt, S. Deser and C. W. Misner, Canonical variables for general relativity, Phys. Rev. 117 (1960) 1595-1602.

[81] R. Arnowitt, S. Deser and C. W. Misner, Energy and the Criteria for Radiation in General Relativity, Phys. Rev. 118 (1960) 1100-1104.

[82] R. L. Arnowitt, S. Deser and C. W. Misner, Coordinate invariance and energy expressions in general relativity, Phys. Rev. 122 (1961) 997.

[83] L. F. Abbott and S. Deser, Stability of Gravity with a Cosmological Constant, Nucl. Phys. B195 (1982) 76-96.

[84] M. H. Dehghani, Charged rotating black branes in anti-de Sitter Einstein-Gauss-Bonnet gravity, Phys. Rev. D67 (2003) 064017, [hep-th/0211191].

[85] M. H. Dehghani and R. B. Mann, Thermodynamics of rotating charged black branes in third order Lovelock gravity and the counterterm method, Phys. Rev. D73 (2006) 104003, [hep-th/0602243]. 